\title{
Impact of circulating SARS-CoV-2 variants on mRNA vaccine-induced immunity
}

https://doi.org/10.1038/s41586-021-04085-y

Received: 10 July 2021

Accepted: 30 September 2021

Published online: 11 October 2021

Check for updates

\author{
Carolina Lucas ${ }^{1,16}$, Chantal B. F. Vogels ${ }^{2,16}$, Inci Yildirim ${ }^{3,4,16}$, Jessica E. Rothman' ${ }^{2}$ Peiwen Lu', \\ Valter Monteiro', Jeff R. Gehlhausen ${ }^{1,5}$, Melissa Campbell ${ }^{6}$, Julio Silva', \\ Alexandra Tabachnikova', Mario A. Peña-Hernandez', M. Catherine Muenker², \\ Mallery I. Breban' ${ }^{2}$, Joseph R. Fauver ${ }^{2}$, Subhasis Mohanty, ${ }^{1,6}$, Jiefang Huang ${ }^{1,6}$, Yale \\ SARS-CoV-2 Genomic Surveillance Initiative*, Albert C. Shaw ${ }^{1,6}$, Albert I. Ko ${ }^{2,6}$, \\ Saad B. Omer ${ }^{2,4,6,17}$, Nathan D. Grubaugh ${ }^{2,7,17}$ \& Akiko Iwasaki ${ }^{1,8,17 凶}$
}

\begin{abstract}
The emergence of SARS-CoV-2 variants with mutations in major neutralizing antibody-binding sites can affect humoral immunity induced by infection or vaccination $^{1-6}$. Here we analysed the development of anti-SARS-CoV-2 antibody and $T$ cell responses in individuals who were previously infected (recovered) or uninfected (naive) and received $m$ RNA vaccines to SARS-CoV-2. While individuals who were previously infected sustained higher antibody titres than individuals who were uninfected post-vaccination, the latter reached comparable levels of neutralization responses to the ancestral strain after the second vaccine dose. T cell activation markers measured upon spike or nucleocapsid peptide in vitro stimulation showed a progressive increase after vaccination. Comprehensive analysis of plasma neutralization using 16 authentic isolates of distinct locally circulating SARS-CoV-2 variants revealed a range of reduction in the neutralization capacity associated with specific mutations in the spike gene: lineages with E484K and N501Y/T (for example, B.1.351 and P.1) had the greatest reduction, followed by lineages with L452R (for example, B.1.617.2). While both groups retained neutralization capacity against all variants, plasma from individuals who were previously infected and vaccinated displayed overall better neutralization capacity than plasma from individuals who were uninfected and also received two vaccine doses, pointing to vaccine boosters as a relevant future strategy to alleviate the effect of emerging variants on antibody neutralizing activity.
\end{abstract}

The ongoing evolution and emergence of SARS-CoV-2 variants raise concerns about the effectiveness of monoclonal antibody therapies and vaccines. The mRNA-based vaccines Pfizer-BioNTech BNT162b2 and Moderna mRNA-1273 encode a stabilized full-length SARS-CoV-2 spike ectodomain derived from the Wuhan-Hu-1 genetic sequence and elicit potent neutralizing antibodies (NAbs) ${ }^{7,8}$. However, emerging SARS-CoV-2 variants with mutations in the spike gene, especially in NAb-binding sites, have been associated with increased transmissibility ${ }^{9,10}$ as well as neutralization resistance to monoclonal antibodies, convalescent plasma and sera from vaccinated individuals ${ }^{1-6}$. To better understand how immune responses triggered by vaccination and/or SARS-CoV-2 infection fare against emerging virus variants, we assembled a cohort of mRNA-vaccinated individuals, previously infected or not, and characterized virus-specific immunological profiles. We examined the effect of SARS-CoV-2 variants containing many different key spike gene mutations in mRNA-vaccinated
individualsusingacomprehensivesetoffull-lengthauthenticSARS-CoV-2 isolates.

\section{Vaccine-induced antibody responses}

First, to characterize SARS-CoV-2-specific adaptive immune responses after mRNA COVID-19 vaccines (Moderna or Pfizer), 40 healthcare workers (HCWs) from the Yale-New Haven Hospital were enrolled in this study between November 2020 and January 2021, with a total of 198 samples. We stratified the vaccinated participants based on previous exposure to SARS-CoV-2 into previously infected (recovered) or uninfected (naive) groups. Previous infection was confirmed by reverse-transcription quantitative PCR (RT-qPCR) and SARS-CoV-2 IgG enzyme-linked immunosorbent assay (ELISA). The HCWs received mRNA vaccines, either Pfizer or Moderna, and we followed them longitudinally pre-vaccination and post-vaccination (Fig. 1a). Cohort

1Department of Immunobiology, Yale University School of Medicine, New Haven, CT, USA. ${ }^{2}$ Department of Epidemiology of Microbial Diseases, Yale School of Public Health, New Haven, CT,

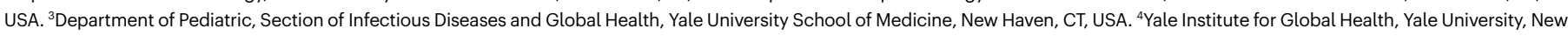
Haven, CT, USA. ${ }^{5}$ Department of Dermatology, Yale University School of Medicine, New Haven, CT, USA. ${ }^{6}$ Department of Medicine, Section of Infectious Diseases, Yale University School of Medicine, New Haven, CT, USA. ${ }^{7}$ Department of Ecology and Evolutionary Biology, Yale University, New Haven, CT, USA. ${ }^{8} \mathrm{Howard}$ Hughes Medical Institute, Chevy Chase, MD, USA. ${ }^{16}$ These

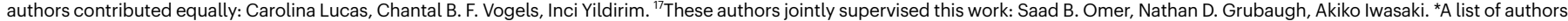
and their affiliations appears at the end of the paper. ${ }^{\circledR e}$-mail: akiko.iwasaki@yale.edu 
a
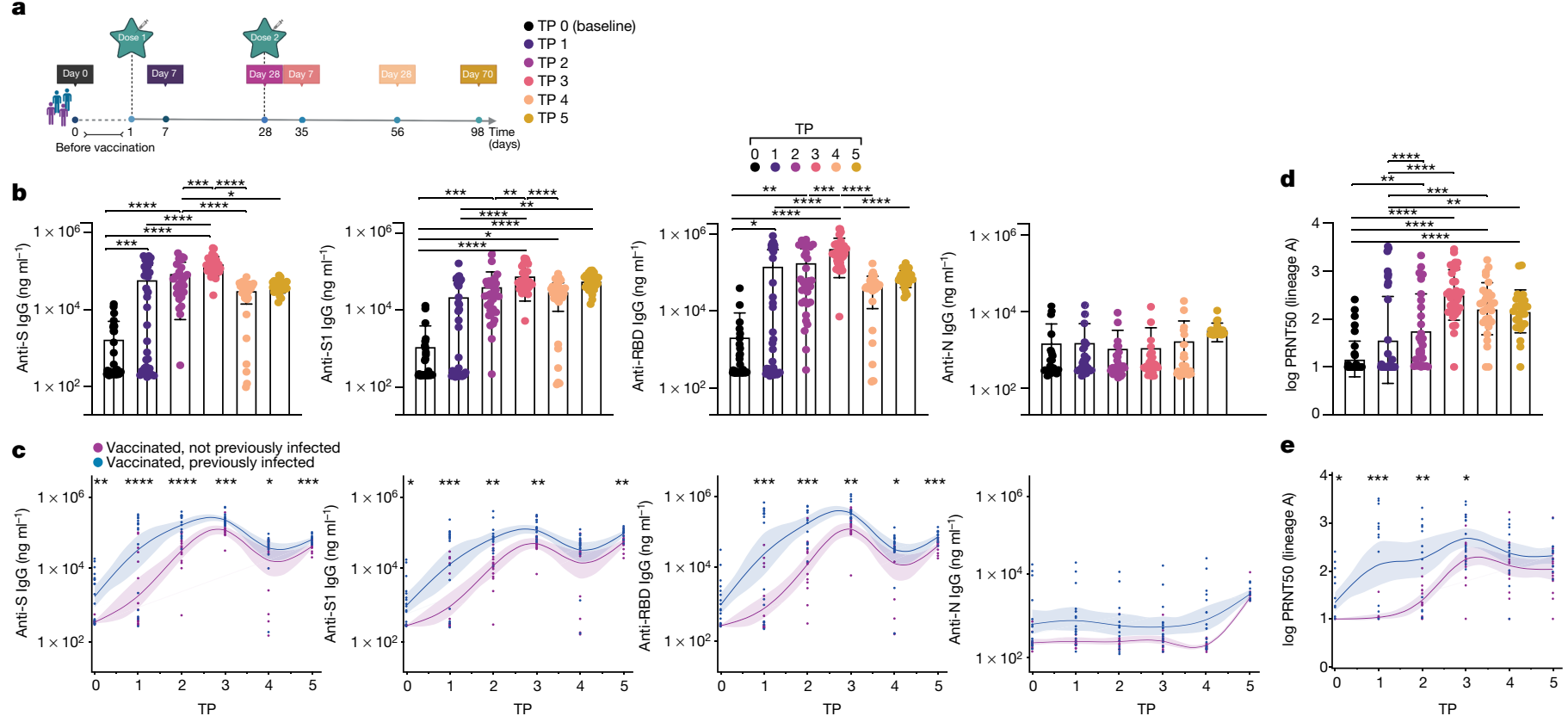

Fig. 1 | Temporal dynamics of anti-SARS-CoV-2 antibodies in vaccinated participants. a, Cohort timeline overview indicated by days after SARS-CoV-2 mRNA vaccination. HCW participants received two doses of the mRNA vaccine and plasma samples were collected as indicated. Baseline (time point 0 (TP 0)), before vaccination; TP 1, 7 days after the first dose; TP 2,28 days after the first dose; TP 3, 7 days after the second dose; TP 4,28 days after the second dose; TP 5,70 days after the second dose. Participants were stratified on the basis of previous exposure toSARS-CoV-2 (purple: vaccinated, uninfected; blue: vaccinated, previously infected). Created with BioRender.com. b, c, Plasma reactivity to the spike (S) protein, receptor-binding domain (RBD) and nucleocapsid $(\mathrm{N})$ measured over time by ELISA. The levels of anti-spike, anti-spike subunit 1 (S1), anti-RBD and anti-nucleocapsid IgG are shown (b). A comparison of the levels of anti-spike, anti-S1, anti-RBD and anti-nucleocapsid IgG in vaccinated participants previously infected or not to SARS-CoV-2 is also displayed (c).d, e, Longitudinal neutralization assay using wild-type SARS-CoV-2, ancestral strain (WA1, USA). The neutralization titre (PRNT50) over time (d), and the plasma neutralization capacity between vaccinated participants previously infected or not to SARS-CoV-2 (e) are shown. In b and d, significance was assessed by one-way analysis of variance (ANOVA) corrected for multiple comparisons using Tukey's method. The bars represent mean values \pm standard deviations. In $\mathbf{c}$ and $\mathbf{e}$, longitudinal data are plotted over time continuously. Regression lines are shown as blue (previously infected) and purple (uninfected). The lines indicate cross-sectional averages from each group with shading representing $95 \% \mathrm{CI}$ and are coloured accordingly. Significance was assessed using unpaired two-tailed $t$-test. TP 0: $n=37$; TP1: $n=35$; TP 2: $n=30 ;$ TP 3: $n=34 ; \operatorname{TP} 4: n=31$ TP 5: $n=27$ ). Each dot represents a single individual. ${ }^{* * *} P<0.0001,{ }^{* * *} P<0.001,{ }^{* *} P<0.01$ and ${ }^{*} P<0.05$. demographics, vaccination status and serostatus are summarized in Extended Data Table 1. We collected plasma and peripheral blood mononuclear cells (PBMCs) sequentially in five time points that covered a period of 98 days after the first vaccination dose. Samples were collected at baseline (before vaccination), 7 and 28 days after the first vaccination dose, and 7,28 and 70 days after the second vaccination dose (Fig. 1a). We determined antibody profiles, using both ELISA and neutralization assays, and assessed cellular immune response, profiled by flow cytometry using frozen PBMCs.

We found that mRNA vaccines induced high titres of virus-specific antibodies that declined over time, as previously reported ${ }^{6,11}$ (Fig. 1b,c). After the first vaccine dose, over $97 \%$ of vaccinated participants developed virus-specific IgG titres, which increased to $100 \%$ after the second dose. IgG titres against the spike protein, spike subunit 1 and receptor-binding domain (RBD) peaked 7 days after the second dose of the vaccine (Fig. 1b, c). No differences were observed in antibody levels between vaccinated participants of different sexes and after stratification by age (Extended Data Fig. 1a). Consistent with previous reports ${ }^{7,12}$, we found that virus-specific IgG levels were significantly higher in the vaccinated group who were previously infected than in the vaccinated group who were uninfected (Extended Data Fig. 1b, c). As expected, given the absence of sequences encoding nucleocapsid antigens in the mRNA vaccines, anti-nucleocapsid antibody titres remain stable over time for vaccinated individuals who were previously infected, and were not affected by vaccination in both the uninfected and the previously infected groups (Fig. 1b, c). We next assessed plasma neutralization activity longitudinally against an authentic SARS-CoV-2 strain
USA-WA1/2020 (lineage A), with a similar spike gene amino acid sequence as the Wuhan-Hu-1 sequence that was used for the mRNA vaccine design, by a 50\% plaque-reduction neutralization (PRNT50) assay. Neutralization activity directly correlated with anti-spike and anti-RBD antibody titres, also peaking at 7 days after the second dose of the vaccine (Fig.1d, e). However, both groups displayed similar neutralization titres against the lineage A virus isolate at the peak of response (Fig. 1d, e). Our data indicate that despite faster and more exuberant antibody responses to viral proteins by vaccinated individuals who were previously infected than vaccinated individuals who were uninfected, vaccination led to overall similar levels of NAbs after the second dose of the vaccine.

\section{Vaccine-induced $T$ cell responses}

A robust $\mathrm{T}$ cell response has also been linked to efficient protective immunity against SARS-CoV-2 (refs. ${ }^{13-15}$ ). Hence, we next longitudinally analysed spike-reactive and nucleocapsid-reactive T cell responses in vaccinated individuals. To detect low-frequency peptide-specific T cell populations, we first expanded antigen-specific $T$ cells by stimulating PBMCs from vaccinated individuals with spike and nucleocapsid peptide pools ex vivo for 6 days, followed by restimulation with the same peptide pools and analysis of activation markers after $12 \mathrm{~h}$. To cover the entire spike protein, two peptide pools (S-I and S-II) were used, while a single peptide pool was used for nucleocapsid stimulation. Spike-reactive $\mathrm{CD} 4^{+}$and $\mathrm{CD} 8^{+} \mathrm{T}$ cells increased over time following vaccination (Fig. 2a, b), as evidenced by an increase in the number of cells expressing the activation markers CD38 and HLA-DR; no differences 


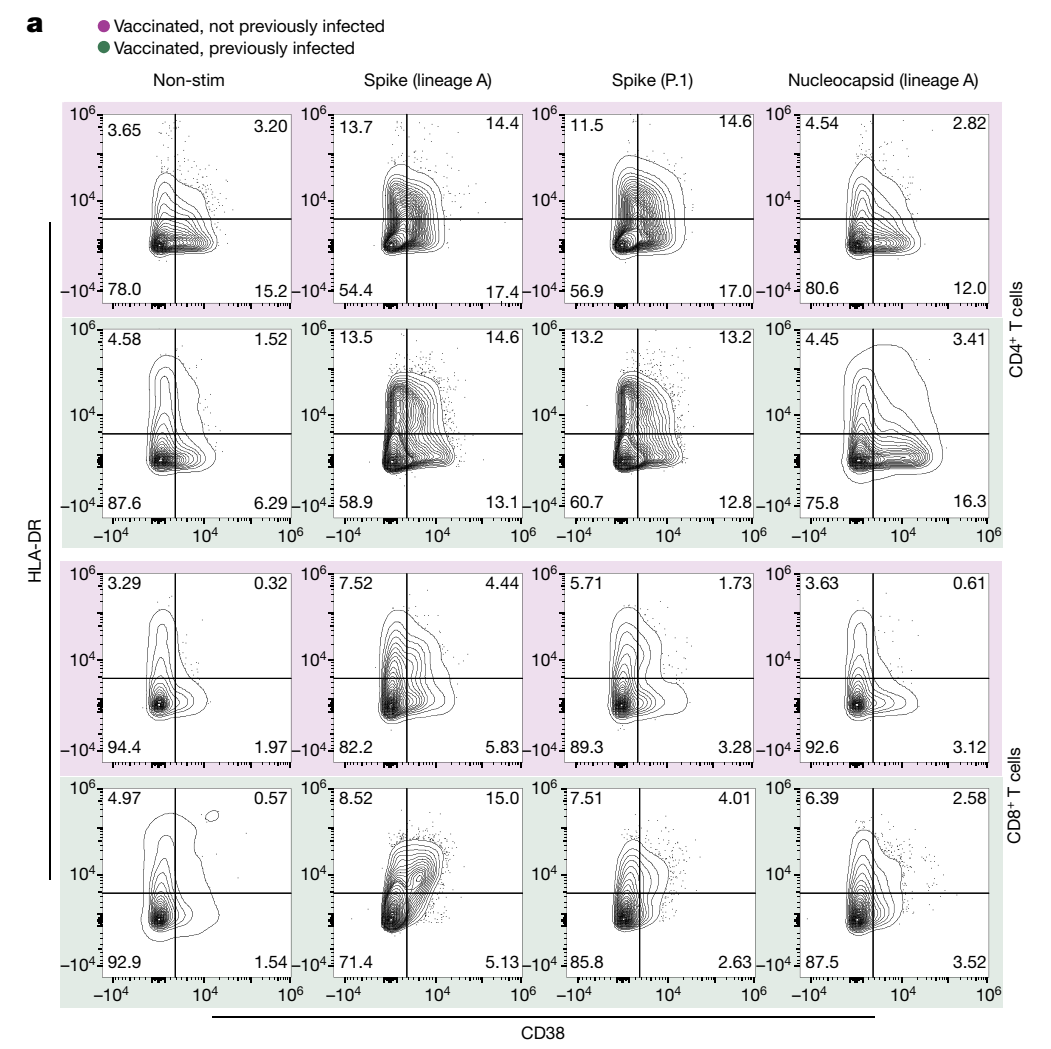

b Vaccinated, not previously infected
Vaccinated, previously infected
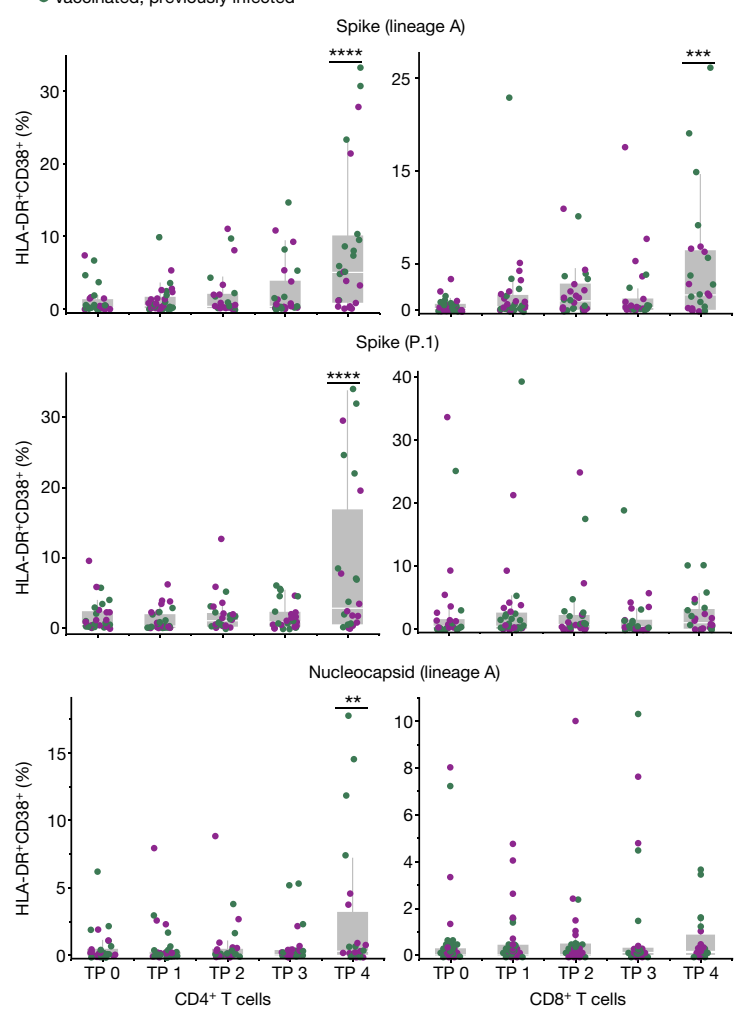

Fig. 2 | Temporal dynamics of anti-SARS-CoV-2 $\mathrm{T}$ cell immunity in vaccinated participants. SARS-CoV-2S-reactive $\mathrm{CD} 4^{+}$and $\mathrm{CD} 8^{+} \mathrm{T}$ cells after in vitro stimulation with SARS-CoV-2S-I and S-II peptide pools and nucleoprotein peptide pool. a, Representative dot plots from four vaccinated individuals, 28 days after the second dose of the vaccine, showing the percentage of double-positive cells expressing HLA-DR and CD38 out of CD4 ${ }^{+}$ $\mathrm{T}$ cells (top) and CD8 ${ }^{+} \mathrm{T}$ cells (bottom). Individuals who were previously infected to SARS-CoV-2 or uninfected are indicated by blue or purple shades, respectively. b. The percentage of double-positive cells, spike-reactive and nucleocapsid-reactive out of $\mathrm{CD} 4^{+} \mathrm{T}$ cells (top) and $\mathrm{CD} 8^{+} \mathrm{T}$ cells (bottom) over time post-vaccination. Individuals who were previously infected to SARS-CoV-2 or uninfected are indicated by blue or purple dots, respectively. Each dot represents a single individual. Significance was assessed by one-way ANOVA corrected for multiple comparisons using Dunnett's method. Vaccination time points were compared with baseline. Stimulation values were subtracted from the respective non-stimulation condition. The bars represent the distribution of variables with quartiles and outliers. The horizontal bars indicate mean values. TP 0: $n=30$; TP1: $n=34$; TP 2: $n=27$; TP $3: n=27$; TP 4: $n=24$ ). Non-stim, non-stimulated PBMCs; nucleocapsid, PBMCs stimulated with the SARS-CoV-2 nucleocapsid protein pool derived from the ancestral lineage A virus, WA1, USA; spike, PBMCs stimulated with the SARS-CoV-2 spike protein pool derived from the ancestral strain lineage A, WA1, USA; spike (P.1), PBMCs stimulated with the SARS-CoV-2 spike protein pool derived from the P.1 variant. ${ }^{* * * *} P<0.0001,{ }^{* * *} P<0.001$ and ${ }^{* *} P<0.01$. were observed between the previously infected and uninfected vaccinated groups. Consistent with previous reports ${ }^{16,17}$, spike-reactive CD4 ${ }^{+}$ T cell responses were comparable among full-length lineage $A$ and P.1 virus isolates. By contrast, spike-reactive $\mathrm{CD} 8^{+} \mathrm{T}$ cell responses were only observed to lineage A, and not P.1, 28 days after the second dose of the vaccine, suggesting that spike-specific $\mathrm{CD} 8^{+} \mathrm{T}$ cell responses can be affected by the mutations within the spike gene of SARS-CoV-2 variants (Fig. 2b). As expected, nucleocapsid-reactive T cells induced after stimulation with a nucleocapsid peptide pool derived from the lineage A virus isolate were primarily observed in vaccinated individuals who were previously infected (Fig. 2b). We also observed elevated numbers of nucleocapsid-reactive $\mathrm{CD} 4{ }^{+} \mathrm{T}$ cells in previously infected individuals at 28 days after the second dose of the vaccine, paralleling general activation of $\mathrm{CD}^{+}{ }^{+} \mathrm{T}$ cells (Fig. 2b, Extended Data Fig. 2a). Moreover, we observed increased counts of activated $\mathrm{CD} 4^{+} \mathrm{T}$ cell, follicular helper $T$ cell and antibody-secreting cells 28 days after the booster vaccine (Extended Data Fig. 2). Thus, T cell responses in vaccinated individuals display similar dynamics as antibody responses.

\section{Vaccine-induced $\mathbf{N A b}$ against variants}

To investigate potential differences in NAb escape between the SARS-CoV-2 variants, we analysed the neutralization capacity of plasma samples from vaccinated individuals against a panel of 18 genetically distinct and authentic SARS-CoV-2 isolates. Among the isolates, 16 were from our Connecticut SARS-CoV-2 genomic surveillance programme representing variants from the same geographical region as our HCW cohort ${ }^{18}$. Our variant panel includes representatives of all lineages currently classified as variants of concern (B.1.1.7, B.1.351, P.1 and B.1.617.2) as well as lineages classified as variants of interest (B.1.427, B.1.429, B.1.525, B.1.526 and B.1.617.1) ${ }^{19}$. In addition, we selected lineages with key spike gene mutations (B.1.517 with N501T, and B.1 and R.1 with $\mathrm{E} 484 \mathrm{~K})^{20}$, and included lineage $\mathrm{A}$ as a comparison (Fig. $3 \mathrm{a}$ ). To help deconvolute the effects of individual mutations, we included four different B.1.526 isolates (labelled as B.1.526 ${ }^{\mathrm{a}-\mathrm{d}}$ ) that represent different phylogenetic clades and key spike gene mutations (L452R, S477N and E484K) (Extended Data Fig. 3b), two different B.1.1.7 isolates with (B.1.1. $7^{\mathrm{b}}$ ) and without (B.1.1.7 ${ }^{\mathrm{a}}$; most common) E484K, and two B.1.351 isolates with (B.1.351 ${ }^{\mathrm{b}}$ ) and without (B.1.351 ; most common) L18F (Fig. 3a, Extended Data Table 2). Except lineage A, all isolates (lineages $B, P$ and $R$ ) have the spike gene D614G mutation located in the receptor-binding motif, which has been reported to have a modest effect on vaccine-elicited neutralization ${ }^{21}$. For each isolate, we highlight key spike protein amino acid differences in the antigenic regions of the amino-terminal domain (NTD), the RBD-ACE2 interface and the furin cleavage site (Fig. 3a). A full list of amino acid substitutions 
a

\begin{tabular}{|c|c|c|c|c|c|c|c|c|c|c|c|c|c|}
\hline \multirow[b]{3}{*}{ Lineage } & \multicolumn{11}{|c|}{ Key spike amino acid differences } & & \\
\hline & \multicolumn{3}{|c|}{ NTD } & \multicolumn{5}{|c|}{ RBD } & \multicolumn{3}{|c|}{ RBM FCS } & & \\
\hline & 18 & 69 & 70 & 417 & 452 & 477 & 478 & 484 & 501 & $\overline{614}$ & 681 & & \\
\hline A & $\mathrm{L}$ & $\mathrm{H}$ & v & $\mathrm{K}$ & $\mathrm{L}$ & S & $\mathrm{T}$ & $\mathrm{E}$ & $\mathrm{N}$ & $\mathrm{D}$ & $P$ & 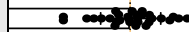 & \\
\hline B.1.526 & & & & & & $N$ & & & & $\mathrm{G}$ & & $=0$ & \\
\hline B.1.526 & & & & & & $\mathrm{N}$ & & & & $\mathrm{G}$ & & 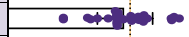 & \\
\hline B.1.1.7a & & $\Delta$ & $\Delta$ & & & & & & $\mathrm{Y}$ & $\mathrm{G}$ & $\mathrm{H}$ & 5. & \\
\hline B.1.517 & & & & & & & & & $T$ & $\mathrm{G}$ & & $\bullet$ & \\
\hline B.1.526 & & & & & & & & $\mathrm{K}$ & & G & & in & \\
\hline B.1.617.2 & & & & & $\mathrm{R}$ & & $\mathrm{K}$ & & & $\mathrm{G}$ & $\mathrm{R}$ & 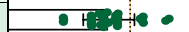 & * \\
\hline R.1 & & & & & & & & $\mathrm{K}$ & & $\mathrm{G}$ & & constion. & * \\
\hline B.1.427 & & & & & $\mathrm{R}$ & & & & & G & & 100 & * \\
\hline B.1.526 & & & & & $\mathrm{R}$ & & & & & G & & 10 & * \\
\hline \begin{tabular}{|l|} 
B.1.429 \\
\end{tabular} & & . & & & $\mathrm{R}$ & & & & & G & & 10 & * \\
\hline \begin{tabular}{|l|} 
B.1.525 \\
\end{tabular} & & $\Delta$ & $\Delta$ & & & & & $\mathrm{K}$ & & G & & 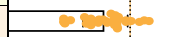 & * \\
\hline B.1.617.1 & & & & & $R$ & & & $\mathrm{Q}$ & & $\mathrm{G}$ & $R$ & $2+10$ & * \\
\hline P.1 & $\mathrm{F}$ & & & & & & & $\mathrm{K}$ & $Y$ & $G$ & & $D^{\prime}=4$ & * \\
\hline B.1 & & & & & & & & $\mathrm{K}$ & $T$ & $G$ & & $\overline{O K}, \infty$ & * \\
\hline B.1.1. $7^{\mathrm{b}}$ & & $\Delta$ & $\Delta$ & & & & & $\mathrm{K}$ & $Y$ & G & $\mathrm{H}$ & 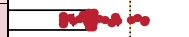 & * \\
\hline B.1.3519 & & & & $\mathrm{N}$ & & & & $\mathrm{K}$ & $\mathrm{Y}$ & G & & $\bullet$ & * \\
\hline B.1.35 b & $F$ & & & $\mathrm{~N}$ & & & & $\mathrm{~K}$ & $Y$ & $\mathrm{G}$ & & ه & * \\
\hline
\end{tabular}

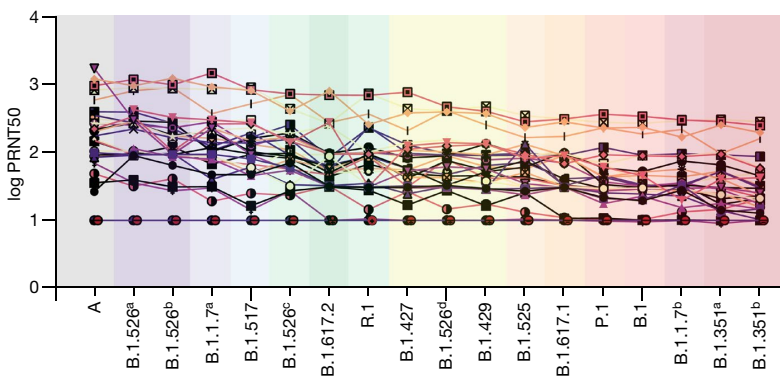

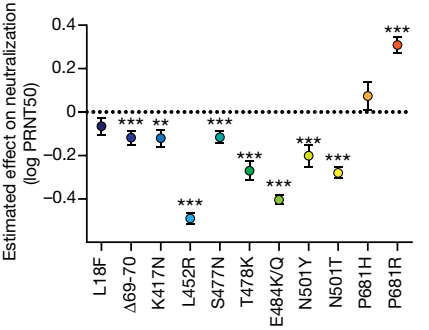

Fig. 3 |Effect of SARS-CoV-2 variants of concern on neutralization capacity of vaccinated participants. a, Plasma neutralization titres against ancestral lineage A virus (WA1, USA) and locally circulating variants. Sixteen SARS-CoV-2 variants were isolated from nasopharyngeal swabs of individuals who were infected and an additional B.1.351 isolate was obtained from BEI. Neutralization capacity was assessed using plasma samples from vaccinated participants, 28 days after the second dose of the SARS-CoV-2 vaccine at the experimental sixfold serial dilutions (from 1:3 to 1:2,430). Key spike mutations within the distinct lineages and plasma neutralization titres (PRNT50) are also shown. Spike mutations are arranged across columns and the rows represent lineages. Significance was assessed by one-way ANOVA corrected for multiple comparisons using Dunnett's method. Neutralization capacity of variants was compared with the neutralization capacity against the ancestral strain. The bars represent mean values \pm standard deviations, and the dotted line indicates the mean value of PRNT50 to the ancestral strain. Superscripted letters indicate different isolates belonging to the same lineage, with distinct spike amino acid changes. $n=32$. FCS, furin cleavage site; RBM, receptor-binding motif. b. Estimated effect of individual mutations on plasma neutralization titres. Neutralization estimates (log PRNT50) and significance were tested with a linear mixed model with subject-level random effects. The dots represent the ratio of linear mixed model coefficients plus intercept to the intercept alone, the error bars represent the standard error, and the dotted line indicates no estimated effect on neutralization. c, Individual trajectories of plasma neutralization titres (PRNT50). Each line represents a single individual. $n=32$. Variants were grouped giving specific spike mutations and are coloured accordingly. Superscripted letters indicate different isolates belonging to the same lineage, with distinct spike amino acid changes. ${ }^{* * * *} P<0.0001$, ${ }^{* * *} P<0.001{ }^{* *} P<0.01$ and ${ }^{*} P<0.05$. and deletions from all genes is provided in Extended Data Table 2. We used a PRNT50 assay to determine the neutralization titres of plasma collected 28 days after the second dose of the vaccine.

When comparing vaccine-induced neutralization against the different isolates in comparison to the lineage A virus isolate, we observed significantly reduced PRNT50 titres for 12 out of the 17 isolates, and the rank order of reduced neutralization mostly clustered by key spike gene amino acid differences (Fig. 3a). Virus isolates with both the E484K and the N501Y (or N501T) mutations (B.1.351 ${ }^{\mathrm{b}}, \mathrm{B} .1 .351^{\mathrm{a}}, \mathrm{B}^{\mathrm{B}} 1.1 .7^{\mathrm{b}}$, B.1 and P.1) reduced neutralization the most (4.6-6.0-fold decrease in PRNT50 titres). Virus isolates with the L452R mutation (B.1.617.1, B.1.429, B.1.526 ${ }^{\mathrm{b}}, \mathrm{B} .1 .427$ and B.1.617.2) were in the next grouping of decreased neutralization (2.5-4.1-fold decrease), which partially overlapped with isolates with E484K but without N501Y/T (B.1.525, R.1 and B.1.526 $; 2.0$-3.8-fold decrease). To further assess the effect of individual mutations, we constructed a linear mixed model with subject-level random effects to account for the differences in neutralization outcome (log-transformed PRNT50 titres) by each individual mutation as compared with lineage A (with no mutation) (Fig. 3b). From our model, we estimated that 8 of the 11 key spike gene mutations that we investigated had significant negative effects on neutralization, and that L452R (2.8-fold decrease in PRNT50 titres; $P<2 \times 10^{-16}$ ) and E484K/Q (2.0-fold decrease; $P<2 \times 10^{-16}$ ) had the greatest individual effects. As combinations of mutations can alter effects differently than the added value of each individually (that is, epistatic interactions), we also created a second linear mixed model that controlled for all of the individual mutations in the first model as well as three common combinations of key spike gene mutations found in our isolates: $\Delta H 69 / \mathrm{V} 70$ and E484K, L452R and P681R, and E484K and N501Y. These combinations of mutations allowed us to assess whether the contribution of mutations together is synergistic, antagonistic or neither. Our model suggests that the $\Delta \mathrm{H} 69 / \mathrm{V} 70$ and $\mathrm{E} 484 \mathrm{~K}$ combination was synergistic (that is, decreased neutralization more than the added effects of each; $\beta=-0.182 ; P=0.005$ ), L452R and P681R was antagonistic (that is, 

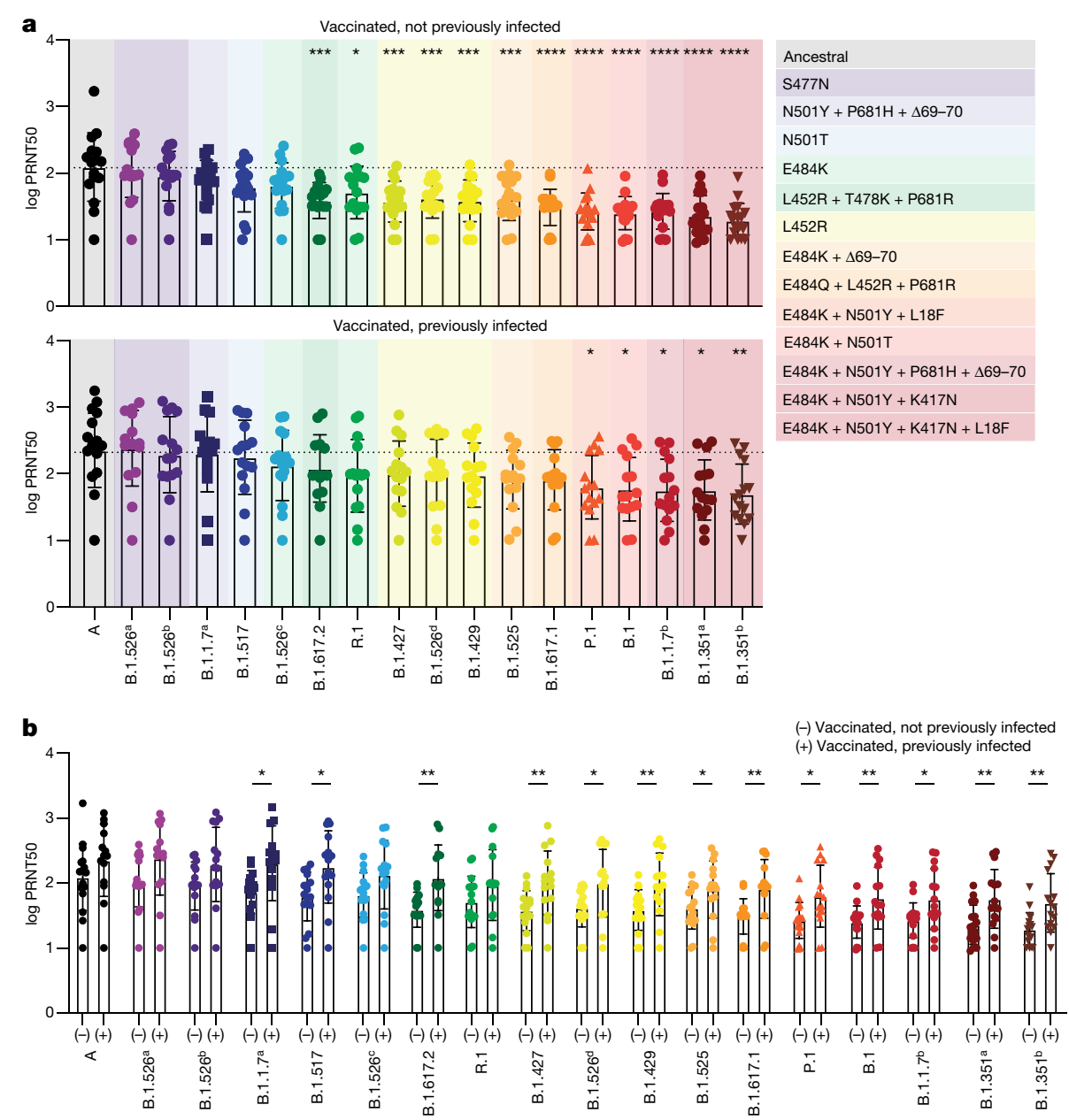

Fig. 4 | Comparison of neutralizing activity in vaccinated $\mathrm{HCWs}$ who were previously infected or not to SARS-CoV-2. Plasma neutralization titres against ancestral lineage A virus (WA1, USA) and locally circulating variants of concern or interest, and other lineages. Sixteen SARS-CoV-2 variants were isolated from nasopharyngeal swabs of individuals who were infected and an additional B.1.351 isolate was obtained from BEI. Neutralization capacity between vaccinated participants who were previously infected or not with SARS-CoV-2 is also shown, and was accessed using plasma samples from vaccinated participants, 28 days after the second dose of the SARS-CoV-2 vaccine at the experimental sixfold serial dilutions (from 1:3 to 1:2,430). a, Neutralization titre among vaccinated individuals. Significance was assessed by one-way ANOVA corrected for multiple comparisons using Dunnett's method. Neutralization capacity to the variants was compared with the neutralization capacity against the ancestral strain. The bars represent mean values \pm standard deviations; the dotted line indicates the mean value of PRNT50 to the ancestral strain. Variants were grouped giving specific spike mutations and are coloured accordingly. $\mathbf{b}$, Comparison of the neutralization titre among vaccinated participants who were previously infected or not to SARS-CoV-2. Significance was accessed using unpaired two-tailed $t$-test. The bars represent mean values \pm standard deviations. (-) Vaccinated, uninfected: $n=17 ;(+)$ Vaccinated, previously infected: $n=15$. Each dot represents a single individual. ${ }^{* * *} P<0.0001,{ }^{* * *} P<0.0011^{* *} P<0.01$ and ${ }^{*} P<0.05$.

\section{Effect of previous infection on NAbs}

To further understand the underlying factors that determine the levels of neutralization activity, we separated individuals by their previous SARS-CoV-2 infection status (that is, previously infected versus uninfected) and determined their neutralization titres to our panel of SARS-CoV-2 isolates. Previous infection occurred between April and December 2020, and for 3 out of 15 HCWs, we were able to identify the lineage of the virus, which were all B.1.3. While the rank order of virus isolates affecting neutralization remained mostly the same, we found that the plasma from vaccinated individuals who were previously infected generally had higher PRNT50 titres against the panel of SARS-CoV-2 isolates than vaccinated individuals who were uninfected (Fig. 4a, b). With the exception of virus isolates from lineages A, B.1.526 ${ }^{\mathrm{a}-\mathrm{c}}$ and R.1, which affected neutralization the least, all other assayed isolates had a significantly higher NAb response in vaccinated individuals who were previously infected (Fig. 4b); only virus isolates with the E484K and $\mathrm{N} 501 \mathrm{Y} / \mathrm{T}$ mutations still significantly reduced neutralization (Fig. 4a). 
For example, the lineage B.1.351 ${ }^{\mathrm{b}}$ isolate (E484K and N501Y) decreased neutralization titres by 13.2 -fold (compared with lineage $A$ ) in vaccinated individuals who were uninfected and by 3.7 -fold in vaccinated individuals who were previously infected, whereas B.1.617.2 (L452R) went from 6.9-fold to 1.5-fold, and B.1.1.7 ${ }^{\mathrm{a}}$ (N501Y) went from 3.4-fold to 0.8 -fold decrease in individuals who were uninfected to individuals who were infected, respectively (Fig. 4a). Thus, our data suggest that plasma neutralization activity against SARS-CoV-2 variants is improved in vaccinated individuals who were previously infected with the virus.

\section{Discussion}

Human NAbs against SARS-CoV-2 can be categorized as belonging to four classes on the basis of their target regions on the RBD. Although the RBD is immunodominant, there is evidence for a substantial role of other spike regions in antigenicity, most notably the NTD supersite $^{22-24}$. These antibodies target epitopes that are closely associated with NTD and RBD residues L18 and $\Delta H 69 / \mathrm{V} 70$, and K417, L452, S477, T478, E484 and N501. Previous studies using pseudovirus constructs reported a significant effect of single spike gene amino acid substitutions, including S477N and E484K located at the RBD-ACE2 interface, in the neutralization activity of plasma from vaccinated individuals $\mathrm{s}^{1-5,25}$.

Using a large panel of genetically diverse authentic SARS-CoV-2 isolates, we found that lineages with $\mathrm{E} 484 \mathrm{~K}$ and N501Y/T led to the most severe decreases in mRNA vaccine-induced neutralization (more than tenfold in vaccinated individuals who were previously uninfected). This group includes B.1.351 (Beta) and P.1 (Gamma), further supporting their importance in regards to vaccines. We also found that a generic lineage B.1 isolate with E484K and N501T, and a rare B.1.1.7 (Alpha) isolate with E484K (also with the common N501Y mutation) have similar effects on neutralization as B.1.351 and P.1. While the combinations of mutations in the B.1 and B.1.1.7 with E484K isolates probably do not increase transmissibility, the additive effects of these two mutations support that surveillance programmes should track all viruses with E484K and $\mathrm{N} 501 \mathrm{Y} / \mathrm{T}$ mutations in addition to variants of concern and/or interest.

While we estimate that the L452R mutation has the greatest individual effect on neutralization, lineages with this mutation, including B.1.617.2 (Delta), are less concerning for NAb escape than the E484K$\mathrm{N} 501 \mathrm{Y} / \mathrm{T}$ combination. From our data, we expect that most fully vaccinated individuals will be protected against B.1.617.2, and that the rise in vaccine-breakthroughs associated with this variant are more probably associated with its high transmissibility ${ }^{26-29}$.

The discrepancies of our results compared with other studies, including ref. ${ }^{6}$, may point to the importance of using fully intact, authentic virus for neutralization assays to detect the effects of epistasis among virus mutations on neutralization assays. Nevertheless, it remains possible that additional factors also contribute to some of the discrepancies between our observations and those of previous studies, including the presence of additional mutations in the membrane and envelope, as well as the composition of our cohorts, predominantly young white women. Differences between cohorts could also account for subtle differences in $\mathrm{T}$ cell responses observed in our study versus the one recently reported by ref. ${ }^{16}$ and ref. ${ }^{17}$. While we observed decreased cross-reactivity of spike-reactive $\mathrm{CD} 8^{+} \mathrm{T}$ cells against $\mathrm{P} .1$ spike peptides, the above studies found that $\mathrm{T}$ cell responses are largely preserved against variants ${ }^{16,17}$. In addition to cohort composition, we used overlapping peptide pools in our assays, and it remains possible that our $T$ cell assay failed to detect under-represented $T$ cell clones affected by variant sequences when sampled in the presence of the majority of conserved peptides. Furthermore, ref. ${ }^{16}$ used an AdV1.26 vaccine, whereas in this study and ref. ${ }^{17}$, mRNA vaccines were used. Overall, our data point to a necessity of active monitoring of $\mathrm{T}$ cell reactivity in the context of SARS-CoV-2 evolution.

The magnitude of the antibody titres in patients with COVID-19 following natural infection has been directly correlated with length of infection and severity ${ }^{30}$. Here we found that vaccinated individuals who were previously infected display an increased resilience in antibody responses against both 'single' and a combination of substitutions in the RBD region, which otherwise severely decreased neutralization activity of vaccinated individuals who were uninfected. Our observations of the effect of pre-existing immunity in vaccinated individuals on their ability to neutralize variants could be explained by the time window between the initial exposure (infection) and vaccination. Moreover, on the basis of timing of previous infection (before the emergence of tested variants) and confirmation by sequencing (three previous infections with B.1.3), we believe that the virus lineage of the infection probably did not have a major effect on our findings. Our observations provide an important rationale for worldwide efforts in characterizing the contribution of pre-existing SARS-CoV-2 immunity to the outcome of various vaccination strategies. Along with recently introduced serological tests ${ }^{31}$, such studies could inform evidence-based risk evaluation, patient monitoring, adaptation of containment methods, and vaccine development and deployment. Finally, these findings suggest that an additional third dose of a vaccine may be beneficial to confer higher protection against SARS-CoV-2 lineages such as B.1.351 and P.1.

\section{Online content}

Any methods, additional references, Nature Research reporting summaries, source data, extended data, supplementary information, acknowledgements, peer review information; details of author contributions and competing interests; and statements of data and code availability are available at https://doi.org/10.1038/s41586-021-04085-y.

1. Cele, S. et al. Escape of SARS-CoV-2 501Y.V2 from neutralization by convalescent plasma. Nature 593, 142-146 (2021).

2. Garcia-Beltran, W. F. et al. Multiple SARS-CoV-2 variants escape neutralization by vaccine-induced humoral immunity. Cell 184, 2372-2383.e9 (2021).

3. Wang, Z. et al. mRNA vaccine-elicited antibodies to SARS-CoV-2 and circulating variants Nature 592, 616-622 (2021).

4. Chen, R. E. et al. Resistance of SARS-CoV-2 variants to neutralization by monoclonal and serum-derived polyclonal antibodies. Nat. Med. 27, 717-726 (2021).

5. Wang, P. et al. Antibody resistance of SARS-CoV-2 variants B.1.351 and B.1.1.7. Nature 593, 130-135 (2021).

6. Stamatatos, L. et al. mRNA vaccination boosts cross-variant neutralizing antibodies elicited by SARS-CoV-2 infection. Science 372, 1413-1418 (2021).

7. Jackson, L. A. et al. An mRNA vaccine against SARS-CoV-2-preliminary report. N. Engl. J. Med. 383, 1920-1931 (2020).

8. Walsh, E. E. et al. Safety and immunogenicity of two RNA-based Covid-19 vaccine candidates. N. Engl. J. Med. 383, 2439-2450 (2020).

9. Davies, N. G. et al. Estimated transmissibility and impact of SARS-CoV-2 lineage B.1.1.7 in England. Science 372, eabg3055 (2021).

10. Sabino, E. C. et al. Resurgence of COVID-19 in Manaus, Brazil, despite high seroprevalence. Lancet 397, 452-455 (2021).

11. Jalkanen, P. et al. COVID-19 mRNA vaccine induced antibody responses against three SARS-CoV-2 variants. Nat. Commun. 12, 3991 (2021).

12. Sahin, U. et al. COVID-19 vaccine BNT162b1 elicits human antibody and TH1 T cell responses. Nature 586, 594-599 (2020).

13. Braun, J. et al. SARS-CoV-2-reactive T cells in healthy donors and patients with COVID-19. Nature 587, 270-274 (2020).

14. Dan, J. M. et al. Immunological memory to SARS-CoV-2 assessed for up to 8 months after infection. Science 371, eabf4063 (2021).

15. Zhao, J. et al. Airway memory $\mathrm{CD}^{+} \mathrm{T}$ cells mediate protective immunity against emerging respiratory coronaviruses. Immunity 44, 1379-1391 (2016)

16. Alter, G. et al. Immunogenicity of Ad26.COV2.S vaccine against SARS-CoV-2 variants in humans. Nature 596, 268-272 (2021).

17. Tarke, A. et al. Impact of SARS-CoV- 2 variants on the total $\mathrm{CD} 4^{+}$and $\mathrm{CD} 8^{+} \mathrm{T}$ cell reactivity in infected or vaccinated individuals. Cell Rep. Med. 2, 100355 (2021).

18. Connecticut SARS-CoV-2 variant surveillance. COVID Tracker https://covidtrackerct.com/ (2020).

19. CDC. SARS-CoV-2 variant classifications and definitions. $C D C$ https://www.cdc.gov/ coronavirus/2019-ncov/variants/variant-info.html (2021).

20. SARS-CoV-2 (hCoV-19) mutation reports. outbreak.info https://outbreak.info/ situation-reports (2021).

21. Zou, J. et al. The effect of SARS-CoV-2 D614G mutation on BNT162b2 vaccine-elicited neutralization. NPJ Vaccines 6, 44 (2021).

22. Robbiani, D. F. et al. Convergent antibody responses to SARS-CoV-2 in convalescent individuals. Nature 584, 437-442 (2020).

23. Barnes, C. O. et al. SARS-CoV-2 neutralizing antibody structures inform therapeutic strategies. Nature 588, 682-687 (2020).

24. McCallum, M. et al. $\mathrm{N}$-terminal domain antigenic mapping reveals a site of vulnerability for SARS-CoV-2. Cell 184, 2332-2347 (2021). 
25. Xie, X. et al. Neutralization of SARS-CoV-2 spike 69/70 deletion, E484K and N501Y variants by BNT162b2 vaccine-elicited sera. Nat. Med. 27, 620-621 (2021).

26. Bernal, J. L. et al. Effectiveness of COVID-19 vaccines against the B.1.617.2 (Delta) variant. N. Engl. J. Med. 385, 585-594 (2021).

27. Bolze, A. et al. Rapid displacement of SARS-CoV-2 variant B.1.1.7 by B.1.617.2 and P.1 in the United States. Preprint at https://doi.org/10.1101/2021.06.20.21259195 (2021).

28. Lustig, Y. et al. Neutralising capacity against Delta (B.1.617.2) and other variants of concern following Comirnaty (BNT162b2, BioNTech/Pfizer) vaccination in health care workers, Israel. Euro Surveill. 26, 2100557 (2021).

29. Challen, R. et al. Early epidemiological signatures of novel SARS-CoV-2 variants: establishment of B.1.617.2 in England. Preprint at https://doi. org/10.1101/2021.06.05.21258365 (2021).

30. Lucas, C. et al. Delayed production of neutralizing antibodies correlates with fatal COVID-19. Nat. Med. 27, 1178-1186 (2021).

31. US Food \& Drug Administration. EUA authorized serology test performance. FDA https:// www.fda.gov/medical-devices/coronavirus-disease-2019-covid-19-emergency-use-autho rizations-medical-devices/eua-authorized-serology-test-performance (2021).

Publisher's note Springer Nature remains neutral with regard to jurisdictional claims in published maps and institutional affiliations. (c) The Author(s), under exclusive licence to Springer Nature Limited 2021

Yale SARS-CoV-2 Genomic Surveillance Initiative

Claire Pearson ${ }^{9}$, Anthony Muyombwe ${ }^{9}$, Randy Downing ${ }^{9}$, Jafar Razeq ${ }^{9}$, Mary Petrone ${ }^{2}$, Isabel Ott ${ }^{2}$, Anne Watkins ${ }^{2}$, Chaney Kalinich ${ }^{2}$, Tara Alpert ${ }^{2}$, Anderson Brito ${ }^{2}$,

Rebecca Earnest ${ }^{2}$, Steven Murphy ${ }^{10}$, Caleb Neal $^{10}$, Eva Laszlo $^{10}$, Ahmad Altajar $^{10}$, Irina Tikhonova", Christopher Castaldi" , Shrikant Mane ${ }^{11}$, Kaya Bilguvar", Nicholas Kerantzas $^{12}$, David Ferguson ${ }^{13}$, Wade Schulz ${ }^{13,14}$, Marie Landry ${ }^{15}$ \& David Peaper ${ }^{15}$

${ }^{9}$ Connecticut State Department of Public Health, Rocky Hill, CT, USA. ${ }^{10}$ Murphy Medical Associates, Greenwich, CT, USA. "Yale Center for Genome Analysis, Yale University, New Haven, CT, USA. ${ }^{12}$ Department of Laboratory Medicine, Yale New Haven Hospital, New Haven CT, USA. ${ }^{13}$ Center for Outcomes Research and Evaluation, Yale New Haven Hospital, New Haven, CT, USA. ${ }^{14}$ Department of Laboratory Medicine, Yale School of Medicine, New Haven, CT, USA. ${ }^{15}$ Departments of Laboratory Medicine and Medicine, Yale University School of Medicine, New Haven, CT, USA. 


\section{Methods}

\section{Ethics statement}

This study was approved by the Yale Human Research Protection Program Institutional Review Board (IRB protocol ID 2000028924). Informed consent was obtained from all enrolled vaccinated HCWs. The IRB from the Yale University Human Research Protection Program determined that the RT-qPCR testing and sequencing of de-identified remnant COVID-19 clinical samples conducted in this study were not research involving human participants (IRB protocol ID 2000028599).

\section{HCW volunteers}

Forty HCW volunteers from the Yale-New Haven Hospital were enrolled and included in this study. The volunteers received the mRNA vaccine (Moderna or Pfizer) between November 2020 and January 2021. Vaccinated donors were stratified in two major groups, previously infected with SARS-CoV-2 (recovered) or uninfected (naive), confirmed by RTqPCR (10 April 2020 to 31 December 2020) and serology (2 April 2020 to $11 \mathrm{March} 2020)$. None of the participants experienced serious adverse effects after vaccination. HCWs were followed serially post-vaccination. Plasma and PBMC samples were collected at baseline (previous to vaccination), 7 and 28 after the first vaccination dose, and 7, 28 and 70 days after the second vaccination dose. Demographic information was aggregated through a systematic review of the electronic health record (EHR) and was used to construct Extended Data Table 1. The clinical data were collected using EPIC EHR May 2020 and REDCap 9.3.6 software. Blood acquisition was performed and recorded by a separate team. Clinical information and time points of collection information of vaccinated $\mathrm{HCW}$ s were not available until after processing and analysing raw data by flow cytometry and ELISA. ELISA, neutralizations and flow cytometry analyses were blinded.

\section{Isolation of plasma and PBMCs}

Whole blood was collected in heparinized CPT blood vacutainers (BDAM362780, BD) and kept on gentle agitation until processing. All blood was processed on the day of collection in a single step standardized method. Plasma samples were collected after centrifugation of whole blood at $600 \mathrm{~g}$ for $20 \mathrm{~min}$ at room temperature without a break. The undiluted plasma was transferred to $15-\mathrm{ml}$ polypropylene conical tubes, and aliquoted and stored at $-80^{\circ} \mathrm{C}$ for subsequent analysis. The PBMC layer was isolated according to the manufacturer's instructions. Cells were washed twice with PBS before counting. Pelleted cells were briefly treated with ACK lysis buffer for 2 min and then counted. Percentage viability was estimated using standard trypan blue staining and an automated cell counter (AMQAX1000, Thermo Fisher). PBMCs were stored at $-80^{\circ} \mathrm{C}$ for subsequent analysis.

\section{SARS-CoV-2-specific antibody measurements}

ELISAs were performed as previously described ${ }^{32}$. In short, Triton X-100 and RNase A were added to serum samples at final concentrations of $0.5 \%$ and $0.5 \mathrm{mg} / \mathrm{ml}$, respectively, and incubated at room temperature for $30 \mathrm{~min}$ before use, to reduce risk from any potential virus in serum. MaxiSorp plates ( 96 wells; 442404 , Thermo Scientific) were coated with $50 \mu \mathrm{l}$ per well of recombinant SARS-CoV-2 STotal (SPN-C52H9-100 $\mu \mathrm{g}$, ACROBiosystems), S1 (S1N-C52H3-100 $\mu \mathrm{g}$, ACROBiosystems), RBD (SPD-C52H3-100 $\mu \mathrm{g}$, ACROBiosystems) and the nucleocapsid protein (NUN-C5227-100 $\mu \mathrm{g}$, ACROBiosystems) at a concentration of $2 \mu \mathrm{g} /$ $\mathrm{ml}$ in PBS and were incubated overnight at $4{ }^{\circ} \mathrm{C}$. The coating buffer was removed, and plates were incubated for $1 \mathrm{~h}$ at room temperature with $200 \mu \mathrm{l}$ of blocking solution (PBS with $0.1 \%$ Tween-20 and $3 \%$ milk powder). Plasma was diluted serially at 1:100, 1:200, 1:400 and 1:800 in dilution solution (PBS with $0.1 \%$ Tween-20 and $1 \%$ milk powder), and $100 \mu \mathrm{l}$ of diluted serum was added for $2 \mathrm{~h}$ at room temperature. Human anti-spike (SARS-CoV-2 human anti-spike (AM006415) (91351, Active Motif) and anti-nucleocapsid SARS-CoV-2 human anti-nucleocapsid
(1A6) (MA5-35941, Active Motif) were serially diluted to generate a standard curve. Plates were washed three times with PBS-T (PBS with $0.1 \%$ Tween-20) and $50 \mu \mathrm{l}$ of HRP anti-human IgG antibody (1:5,000; A00166, GenScript) diluted in dilution solution added to each well. After $1 \mathrm{~h}$ of incubation at room temperature, plates were washed six times with PBS-T. Plates were developed with $100 \mu$ l of TMB Substrate Reagent Set (555214, BD Biosciences) and the reaction was stopped after $5 \mathrm{~min}$ by the addition of $2 \mathrm{~N}$ sulfuric acid. Plates were then read at a wavelength of $450 \mathrm{~nm}$ and $570 \mathrm{~nm}$.

\section{T cell stimulation}

For the in vitro stimulation, PBMCs were stimulated with HLA class I and HLA-DR peptide pools at the concentration of 1-10 $\mathrm{gg} \mathrm{ml}^{-1}$ per peptide and cultured for 7 days. On day 0 , PBMCs were thawed, counted and plated in a total of $5-8 \times 10^{5}$ cells per well in 200 ul of RPMI 1640 medium (Gibco) supplemented with $1 \%$ sodium pyruvate (NEAA), $100 \mathrm{U} / \mathrm{ml}$ penicillin-streptomycin (Biochrom) and $10 \% \mathrm{FBS}$ at $37^{\circ} \mathrm{C}$ and $5 \% \mathrm{CO}_{2}$. On day 1 , cells were washed and the stimulation was performed with: PepMix SARS-CoV-2 spike glycoprotein pool 1 and pool 2 (GenScript), PepMix P.1SARS-CoV-2 spike glycoprotein pool 1 and pool 2 (JPT) and PepMix SARS-CoV-2 nucleocapsid protein (JPT). Stimulation controls were performed with PBS (unstimulated). Peptide pools were used at $1 \mu \mathrm{g} \mathrm{ml}^{-1}$ per peptide. Incubation was performed at $37^{\circ} \mathrm{C}, 5 \% \mathrm{CO}_{2}$ for 6 days. On day 6 , cells were restimulated with $10 \mu \mathrm{g} \mathrm{ml}^{-1}$ per peptide and subsequently incubated for $12 \mathrm{~h}$, with the last $6 \mathrm{~h}$ being in the presence of $10 \mu \mathrm{g} \mathrm{ml}^{-1}$ brefeldin A (Sigma-Aldrich). Following this incubation, cells were washed with PBS 2 mM EDTA and prepared for analysis by flow cytometry.

\section{Flow cytometry}

Antibody clones and vendors were as follows: BB515 anti-hHLA-DR (G46-6, 1:400; BD Biosciences), BV605 anti-hCD3 (UCHT1, 1:300; BioLegend), BV785 anti-hCD19 (SJ25C1, 1:300; BD Biosciences), BV785 anti-hCD4 (SK3, 1:200; BioLegend), APCFire750 or BV711 anti-hCD8 (SK1,1:200; BioLegend), AlexaFluor 700 anti-hCD45RA (HI100,1:200; BD Biosciences), PE anti-hPD1 (EH12.2H7, 1:200; BioLegend), APC or PE-CF594 anti-hTIM3 (F38-2E2, 1:50; BioLegend), BV711 anti-hCD38 (HIT2, 1:200; BioLegend), BB700 anti-hCXCR5 (RF8B2, 1:50; BD Biosciences), PE-CF594 anti-hCD25 (BC96, 1:200; BD Biosciences), AlexaFluor 700 anti-hTNFa (MAb11, 1:100; BioLegend), PE or APC/Fire750 anti-hIFNY (4S.B3, 1:60; BioLegend), FITC anti-hGranzymeB (GB11, 1:200; BioLegend), BV785 anti-hCD19 (SJ25C1,1:300; BioLegend), BV421 anti-hCD138 (MI15, 1:300; BioLegend), AlexaFluor700 anti-hCD20 (2H7, 1:200; BioLegend), AlexaFluor 647 anti-hCD27 (M-T271, 1:350; BioLegend), PE/Dazzle594 anti-hIgD (IA6-2,1:400; BioLegend), Percp/ Cy5.5 anti-hCD137 (4B4-1,1:150; BioLegend) and PE anti-CD69 (FN-50, 1:200; BioLegend), and APC anti-hCD40L (24-31,1:100; BioLegend). In brief, freshly isolated PBMCs were plated at $1-2 \times 10^{6}$ cells per well in a 96-well U-bottom plate. Cells were resuspended in Live/Dead Fixable Aqua (Thermo Fisher) for $20 \mathrm{~min}$ at $4{ }^{\circ} \mathrm{C}$. Following a wash, cells were blocked with Human TruStan FcX (BioLegend) for 10 min at room temperature. Cocktails of desired staining antibodies were added directly to this mixture for $30 \mathrm{~min}$ at room temperature. For secondary stains, cells were first washed and supernatant aspirated; then to each cell pellet, a cocktail of secondary markers was added for $30 \mathrm{~min}$ at $4{ }^{\circ} \mathrm{C}$. Before analysis, cells were washed and resuspended in $100 \mu \mathrm{l} 4 \%$ PFA for $30 \mathrm{~min}$ at $4{ }^{\circ} \mathrm{C}$. Following this incubation, cells were washed and prepared for analysis on an Attune NXT (Thermo Fisher). Data were analysed using FlowJo software version 10.6 software (Tree Star). The specific sets of markers used to identify each subset of cells are summarized in Extended Data Fig. 4.

\section{Cell lines and virus}

TMPRSS2-VeroE6 kidney epithelial cells were cultured in DMEM supplemented with $1 \%$ sodium pyruvate (NEAA) and $10 \% \mathrm{FBS}$ at $37^{\circ} \mathrm{C}$ and $5 \% \mathrm{CO}_{2}$. The cell line was obtained from the American Type Culture 
Collection and tested negative for contamination with mycoplasma. SARS-CoV-2 lineage A (USA-WA1/2020) was obtained from BEI Resources (no. NR-52281) and was amplified in TMPRSS2-VeroE6. Cells were infected at a multiplicity of infection of 0.01 for 3 days to generate a working stock, and after incubation, the supernatant was clarified by centrifugation ( $450 \mathrm{~g}$ for $5 \mathrm{~min}$ ), and filtered through a $0.45-\mu \mathrm{m}$ filter. The pelleted virus was then resuspended in PBS and aliquoted for storage at $-80^{\circ} \mathrm{C}$. Viral titres were measured by standard plaque assay using TMPRSS2-VeroE6. In brief, $300 \mu \mathrm{l}$ of serial fold virus dilutions were used to infect Vero E6 cells in MEM supplemented with $\mathrm{NaHCO}_{3}, 4 \% \mathrm{FBS}$ and $0.6 \%$ Avicel RC-581. Plaques were resolved at $48 \mathrm{~h}$ post-infection by fixing in $10 \%$ formaldehyde for $1 \mathrm{~h}$ followed by $0.5 \%$ crystal violet in $20 \%$ ethanol staining. Plates were rinsed in water to plaques enumeration. All experiments were performed in a biosafety level 3 laboratory with approval from the Yale Environmental Health and Safety office.

\section{SARS-CoV-2 variant sequencing and isolation}

SARS-CoV-2 samples were sequenced as part of the Yale SARS-CoV-2 Genomic Surveillance Initiative's weekly surveillance programme in Connecticut, USA ${ }^{33}$. Lineages were sequenced and isolated as previously described ${ }^{34}$. In brief, nucleic acid was extracted from de-identified remnant nasopharyngeal swabs and tested with our multiplexed RTqPCR variant assay to select samples with a N1 cycle threshold value of 35 or lower for sequencing ${ }^{35,36}$. Libraries were prepared with a slightly adjusted version of the Illumina COVIDSeq Test RUO version. The Yale Center for Genome Analysis sequenced pooled libraries of up to 96 samples on the Illumina NovaSeq (paired-end 150). Data were analysed and consensus genomes were generated using iVar (version 1.3.1) ${ }^{37}$. Variants of interest and concern, lineages with mutations of concern (E484K), as well as other lineages as controls were selected for virus isolation. In total, 16 viruses were isolated belonging to 12 lineages (Extended Data Fig. 3, Extended Data Table 2). In addition, ancestral lineage A virus and lineage B.1.351 virus were obtained from BEI.

Samples selected for virus isolation were diluted 1:10 in DMEM and then filtered through a $45-\mu \mathrm{m}$ filter. The samples were tenfold serially diluted from 1:50 to 1:19,531,250. The dilution was subsequently incubated with TMPRSS2-Vero E6 in a 96-well plate and adsorbed for $1 \mathrm{~h}$ at $37^{\circ} \mathrm{C}$. After adsorption, replacement medium was added, and cells were incubated at $37^{\circ} \mathrm{C}$ for up to 5 days. Supernatants from cell cultures with cytopathic effect were collected, frozen, thawed and subjected to RT-qPCR. Fresh cultures were inoculated with the lysates as described above for viral expansion. Viral infection was subsequently confirmed through reduction of cycle threshold values in the cell cultures with the multiplex variant qPCR assay. Expanded viruses were resequenced following the same method as described above and genome sequences were uploaded to GenBank (Supplementary Data Table 2), and the aligned consensus genomes are available on GitHub (https://github. com/grubaughlab/paper_2021_Nab-variants). Nextclade v1.5.0 (https:// clades.nextstrain.org/) was used to generate a phylogenetic tree (Extended Data Fig. 3) and to compile a list of amino acid changes in the virus isolates as compared with the Wuhan-Hu-1 reference strain (Extended Data Table 2). Key spike amino acid differences were identified based on the outbreak.info mutation tracker ${ }^{20}$.

\section{Neutralization assay}

Sera from vaccinated HCWs were isolated as described before and then heat treated for $30 \mathrm{~min}$ at $56^{\circ} \mathrm{C}$. Sixfold serially diluted plasma, from 1:3 to 1:2,430 were incubated with SARS-CoV-2 variants, for $1 \mathrm{~h}$ at $37^{\circ} \mathrm{C}$. The mixture was subsequently incubated with TMPRSS2-VeroE6 in a 12-well plate for $1 \mathrm{~h}$, for adsorption. Then, cells were overlayed with MEM supplemented with $\mathrm{NaHCO}_{3}, 4 \% \mathrm{FBS}$ and $0.6 \%$ Avicel mixture. Plaques were resolved at $40 \mathrm{~h}$ post-infection by fixing in $10 \%$ formaldehyde for $1 \mathrm{~h}$ followed by staining in $0.5 \%$ crystal violet. All experiments were performed in parallel with sera from baseline controls, in an established viral concentration to generate 60-120 plaques per well.

\section{Statistical analysis}

All analyses of patient samples were conducted using GraphPad Prism 8.4.3,JMP15 and R3.4.3. Multiple group comparisons were analysed by running parametric (ANOVA) statistical tests. Multiple comparisons were corrected using Tukey's and Dunnett's tests as indicated in the figure legends. For the comparison between stable groups, two-sided unpaired $t$-test was used for the comparison. The effect of spike mutations was assessed using a linear mixed model with an outcome of log-transformed PRNT50 and random effects accounting for each individual participant. This was done using the 'Ime4' package in R 4.0.1 (ref. ${ }^{38}$ ).

\section{Reporting summary}

Further information on research design is available in the Nature Research Reporting Summary linked to this paper.

\section{Data availability}

The data generated during the current study are available as indicated. All of the background information for HCW participants and data generated in this study are included in the Supplementary Data file. All of the genome information for SARS-CoV-2 variants used in this study are available in Extended Data Table 2, and the aligned consensus genomes are available on GitHub (https://github.com/grubaughlab/ paper_2021_Nab-variants). Additional correspondence and requests for materials should be addressed to the corresponding author (A.I.).

32. Amanat, F. et al. A serological assay to detect SARS-CoV-2 seroconversion in humans. Nat. Med. 26, 1033-1036 (2020).

33. Kalinich, C. C. et al. Real-time public health communication of local SARS-CoV-2 genomic epidemiology. PLoS Biol. 18, e3000869 (2020).

34. Mao, T. et al. A stem-loop RNA RIG-I agonist confers prophylactic and therapeutic protection against acute and chronic SARS-CoV-2 infection in mice. Preprint at bioRxiv https://doi.org/10.1101/2021.06.16.448754 (2021).

35. Vogels, C. B. F. et al. Multiplex qPCR discriminates variants of concern to enhance global surveillance of SARS-CoV-2. PLoS Biol. 19, e3001236 (2021).

36. Vogels, C. B. F., Fauver, J. R. \& Grubaugh, N. D. Multiplexed RT-qPCR to screen for SARS-COV-2 B.1.1.7, B.1.351, and P.1 variants of concern V.3. Protocols https://doi. org/10.17504/protocols.io.br9vm966 (2021).

37. Grubaugh, N. D. et al. An amplicon-based sequencing framework for accurately measuring intrahost virus diversity using PrimalSeq and iVar. Genome Biol. 20, 8 (2019).

38. Bates, D., Mächler, M., Bolker, B. \& Walker, S. Fitting linear mixed-effects models using Ime4. J. Stat. Softw. 67, 1-48 (2015).

Acknowledgements We thank M. Linehan for technical and logistical assistance, D. Mucida for discussions, and M. Suchard and W. Hanage for statistics advice. This work was supported by the Women's Health Research at Yale Pilot Project Program (A.I.), Fast Grant from Emergent Ventures at the Mercatus Center (A.I. and N.D.G.), Mathers Foundation, the Ludwig Family Foundation, the Department of Internal Medicine at the Yale School of Medicine, Yale School of Public Health and the Beatrice Kleinberg Neuwirth Fund. A.I. is an investigator of the Howard Hughes Medical Institute. C.L. is a Pew Latin American Fellow. C.B.F.V. is supported by NWO Rubicon 019.181EN.004.

Author contributions C.L., C.B.F.V., I.Y., S.B.O., N.D.G. and A.I. conceived the study. C.L., J.S., A.T., M.A.P.-H., S.M. and J.H. collected and processed patient PBMC and plasma samples. C.L. and V.M. performed SARS-CoV-2-specific antibody ELISAs. C.L. isolated SARS-CoV-2 variants and performed the neutralization assays. C.L., P.L. and J.R.G. performed the flow cytometry and the flow data analyses. I.Y. and M.C. collected epidemiological and clinical data. C.B.F.V., M.I.B., J.R.F. and Y.S.G.S.N. performed virus sequencing. M.C.M. processed and stored patient specimens. M.C. assisted in the identification and enrolment of volunteers. C.L., C.B.F.V., J.E.R. and N.D.G. analysed the data. C.L., C.B.F.V., N.D.G. and A.I. drafted the manuscript. All authors reviewed and approved the manuscript. N.D.G. and A.I. secured funds and supervised the project.

Competing interests A.I. served as a consultant for Spring Discovery, Boehringer Ingelheim and Adaptive Biotechnologies. I.Y. reported being a member of the mRNA-1273 Study Group and has received funding to her institution to conduct clinical research from BioFire Medlmmune, Regeneron, PaxVax, Pfizer, GSK, Merck, Novavax, Sanofi-Pasteur and Micron. N.D.G. is a consultant for Tempus Labs to develop infectious disease diagnostic assays. All other authors declare no competing interests.

\section{Additional information}

Supplementary information The online version contains supplementary material available at https://doi.org/10.1038/s41586-021-04085-y.

Correspondence and requests for materials should be addressed to Akiko Iwasaki.

Peer review information Nature thanks Pei-Yong Shi and the other, anonymous, reviewer(s) for their contribution to the peer review of this work. Peer review reports are available.

Reprints and permissions information is available at http://www.nature.com/reprints. 


$\begin{array}{llll}\text { A) } & \bullet \text { (0) Baseline } & \bullet \text { (3) } 7 \text { days post } 2 \text { dose } & \text { (-) Vaccinated- Non-previous infected } \\ & \bullet \text { (1) } 7 \text { days post 1dose } & \text { (4) } 28 \text { days post 2dose } & (+) \text { Vaccinated- Previous infected } \\ & \bullet \text { (2) } 28 \text { days post 1dose } & \text { (5) } 70 \text { days post } 2 \text { dose } & \end{array}$
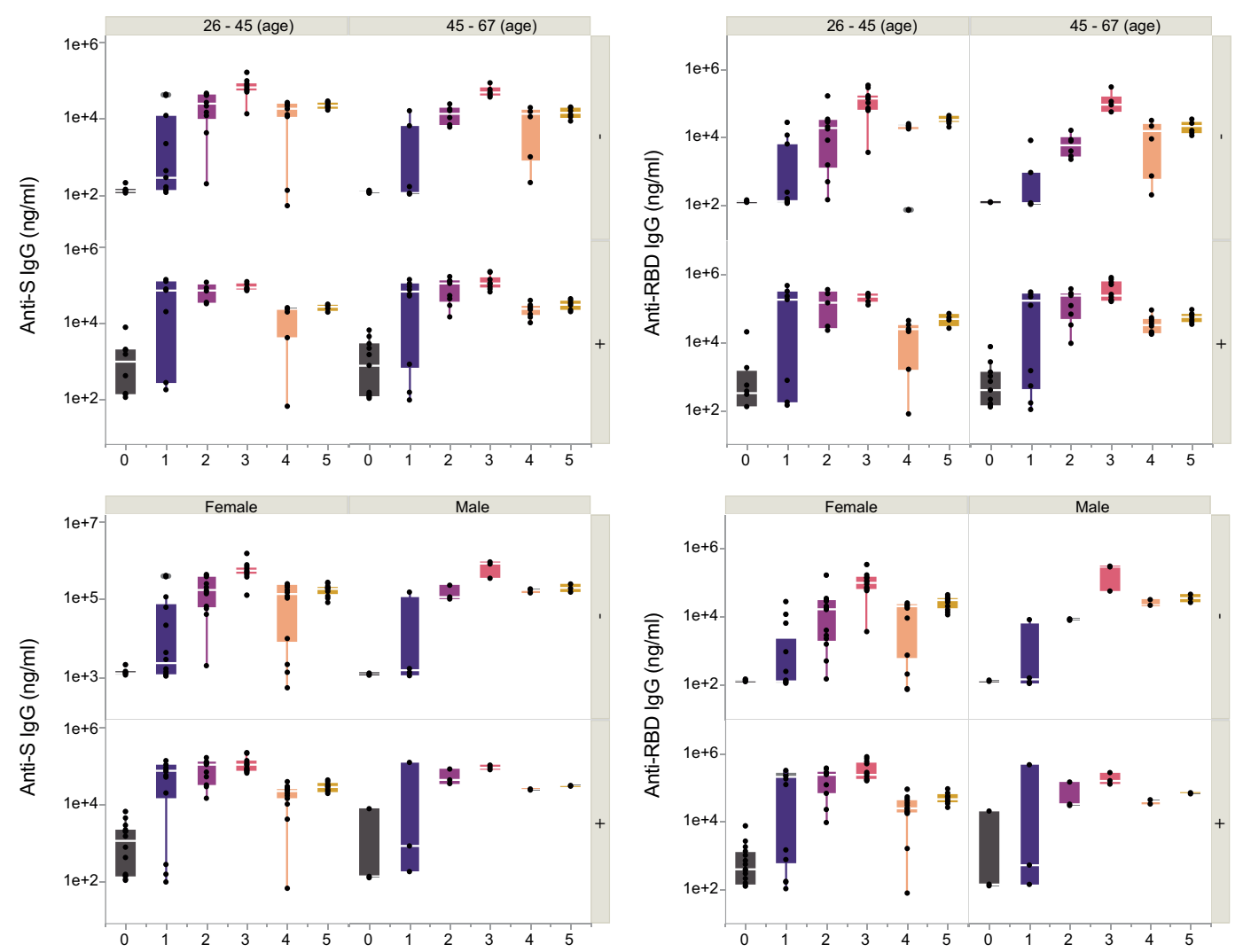

B)
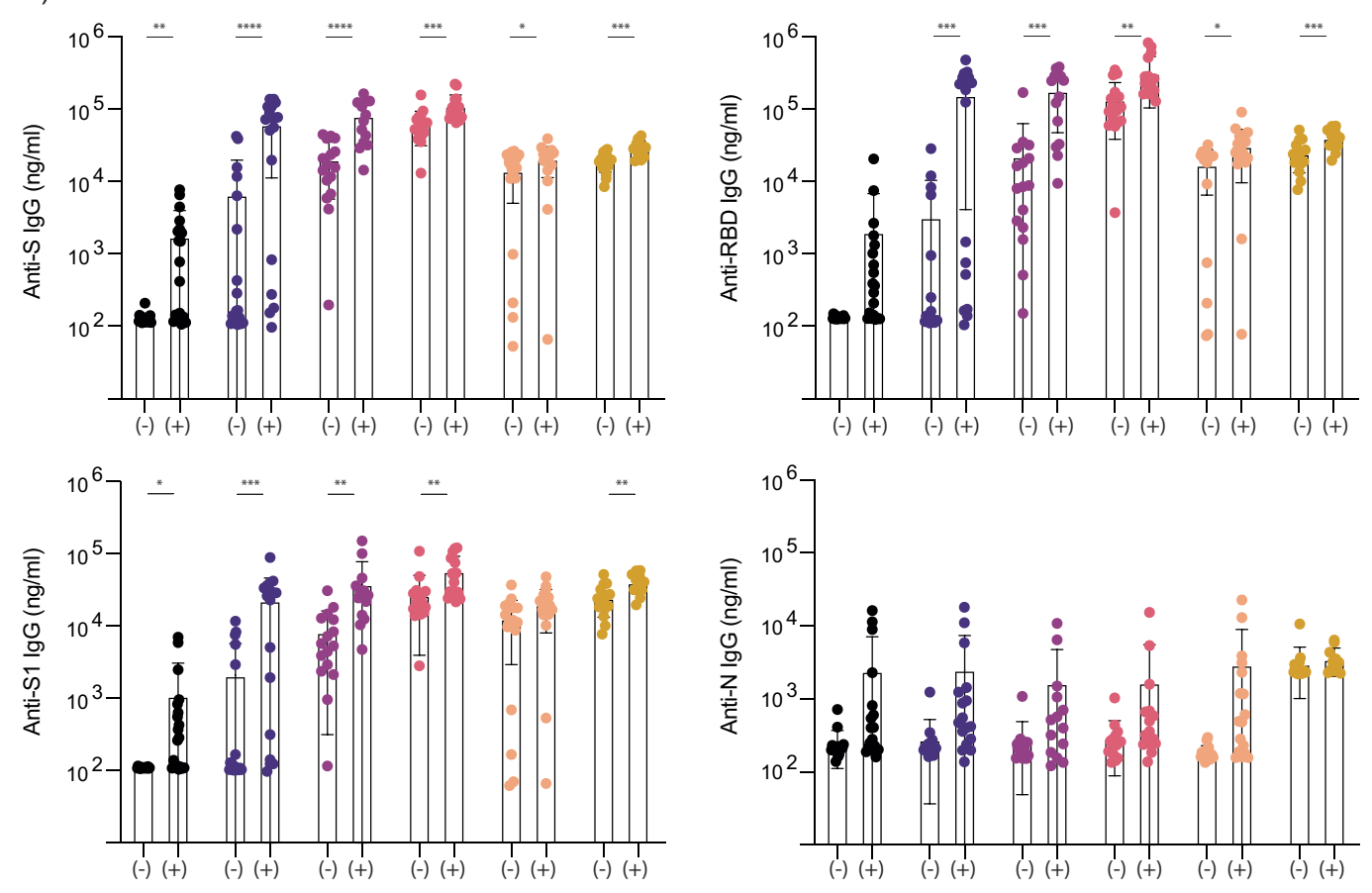

Extended Data Fig. 1 | See next page for caption. 
Extended Data Fig. 1 Correlation of virus-specific antibodies with age and sex of participants. a, b, Plasma reactivity to $S$ protein and $R B D$ in vaccinated participants measured over time by ELISA. HCW participants received 2 doses of the mRNA vaccines and plasma samples were collected as at the indicated time points (TP). Baseline, previously to vaccination; 1 Time point, 7 days post 1 dose; 2 Time point, 28 days post 1 dose; 3 Time point, 7 days post 2 dose; 4 Time point, 28 days post 2 dose; 5 Time point, 70 days post 2 dose. a, Anti-S (left) and Anti-RBD (right) IgG levels stratified by vaccinated participants accordingly to age and sex. Significance was accessed using unpaired two-tailed $t$-test. Boxes represent variables' distribution with quartiles and outliers. Horizontal bars, mean values. b, Anti-S, Anti-S1, Anti-RBD and Anti-N IgG comparison in vaccinated participants previously infected or not to SARS-CoV-2. Longitudinal data plotted over time. Significance was accessed using unpaired two-tailed t-test. Boxes represent mean values \pm standard deviations. TP, vaccination time point. Anti-S IgG (TP0, $n=37 ; T P 1, n=35 ; T P 2, n=30 ; T P 3, n=34 ; T P 4, n=34 ; T P 5$, $\mathrm{n}=28$ ). Anti-S1 IgG (TP0, n=37; TP1, n=35; TP2, $\mathrm{n}=30 ; \mathrm{TP} 3, \mathrm{n}=34 ; \mathrm{TP} 4, \mathrm{n}=34 ; \mathrm{TP} 5$, $\mathrm{n}=27$ ). Anti-RBD IgG (TPO, $\mathrm{n}=37 ; \mathrm{TP} 1, \mathrm{n}=35 ; \mathrm{TP} 2, \mathrm{n}=30 ; \mathrm{TP} 3, \mathrm{n}=34 ; \mathrm{TP} 4, \mathrm{n}=34$; TP5, n=27). Anti-N IgG (TPO, n=37;TP1, n=35;TP2, n=30;TP3, n=34;TP4, n=34; $\mathrm{TP} 5, \mathrm{n}=27)$. S, spike. S1, spike subunit 1 . RBD, receptor binding domain. $\mathrm{N}$, nucleocapsid. Each dot represents a single individual. ${ }^{* * * *} \mathrm{p}<.0001^{* * *} \mathrm{p}<.001$ ${ }^{* *} \mathrm{p}<.01^{*} \mathrm{p}<.05$. 


\section{Article}
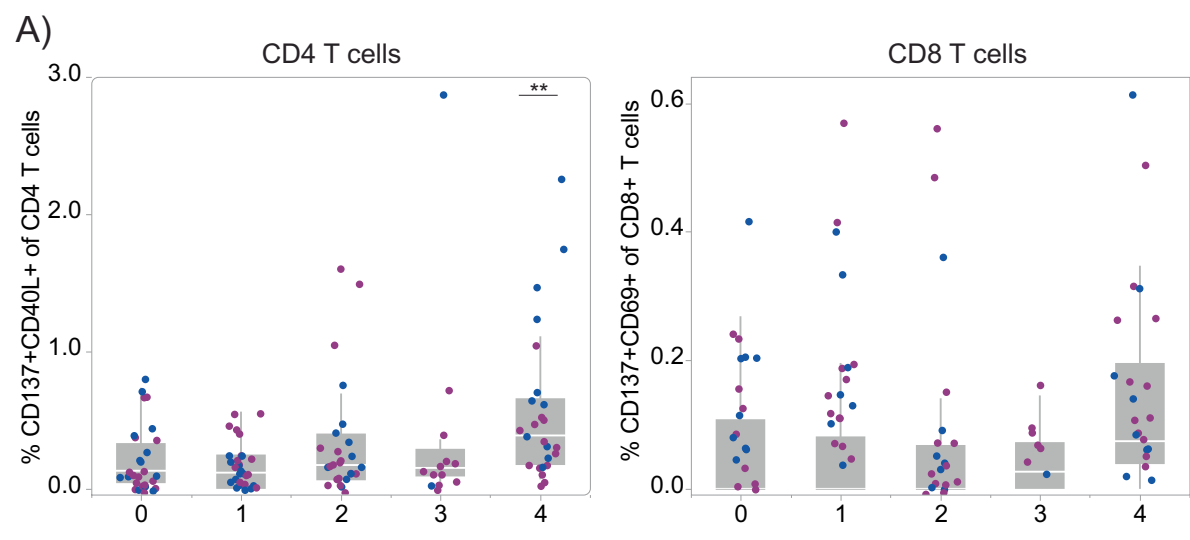

Vaccinated- Non-previous infected

Vaccinated- Previous infected

(0) Baseline

(1) 7 days post 1 dose

(2) 28 days post 1 dose

(3) 7 days post 2 dose

(4) 28 days post 2 dose

B)

Naive B cells
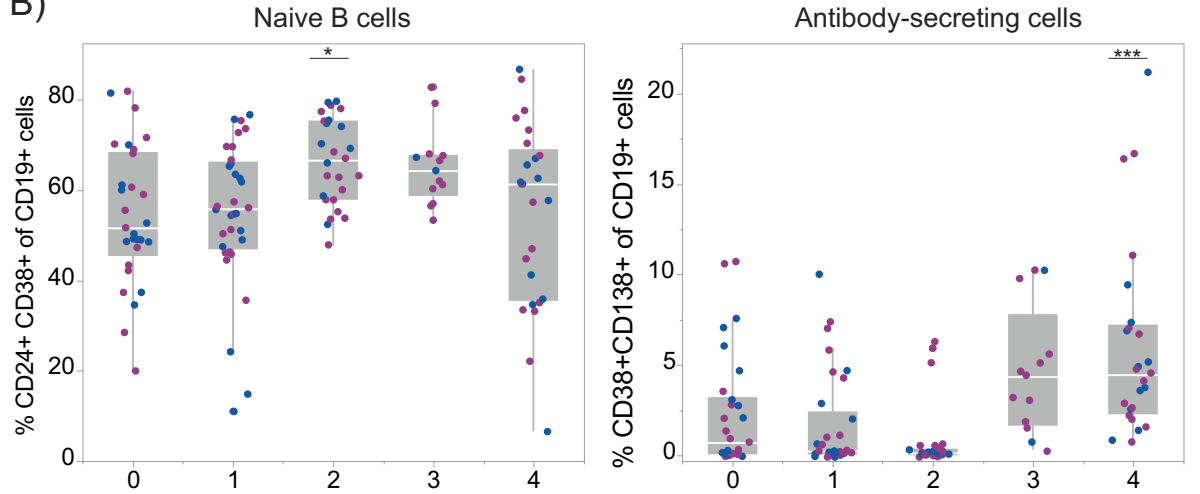

C)

Follicular helper CD4 T cells

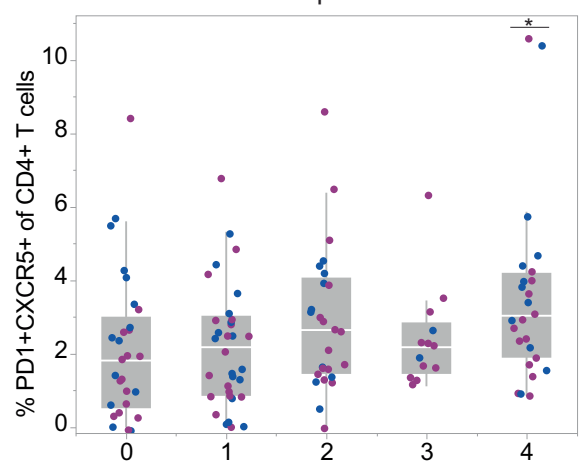

Extended Data Fig. 2 | Cellular immune profiling post SARS-CoV-2

vaccination. a-c, Immune cell subsets of interest, plotted as a percentage of a parent population over time according to the vaccination time points. $\mathrm{HCW}$ participants received 2 doses of the $m R N A$ vaccines and PBMCs samples were collected as at the indicated time points (TP). Baseline, previously to vaccination; 1 Time point, 7 days post 1 dose; 2 Time point, 28 days post 1 dose; 3 Time point, 7 days post 2 dose; 4 Time point, 28 days post 2 dose. Percentage of activated $\mathrm{T}$ cell subsets $(\mathbf{a})$, B cell subsets $(\mathbf{b})$ and Tfh cells $(\mathbf{c})$ among vaccinated

individuals over time. Individuals previously infected to SARS-CoV-2 or uninfected are indicated by blue or purple dots, respectively. Each dot represents a single individual. Significance was assessed by One-way ANOVA corrected for multiple comparisons using Dunnett's method. Vaccination time points were compared with baseline. Boxes represent variables' distribution with quartiles and outliers. Horizontal bars, mean values.TP, vaccination time point (TP0, $n=29 ;$ TP1, n=33; TP2, n=26; TP3, n=13; TP4, n=25). ${ }^{* * *} \mathrm{p}<.0011^{* *} \mathrm{p}<$ $.01 * \mathrm{p}<.05$. 
A)

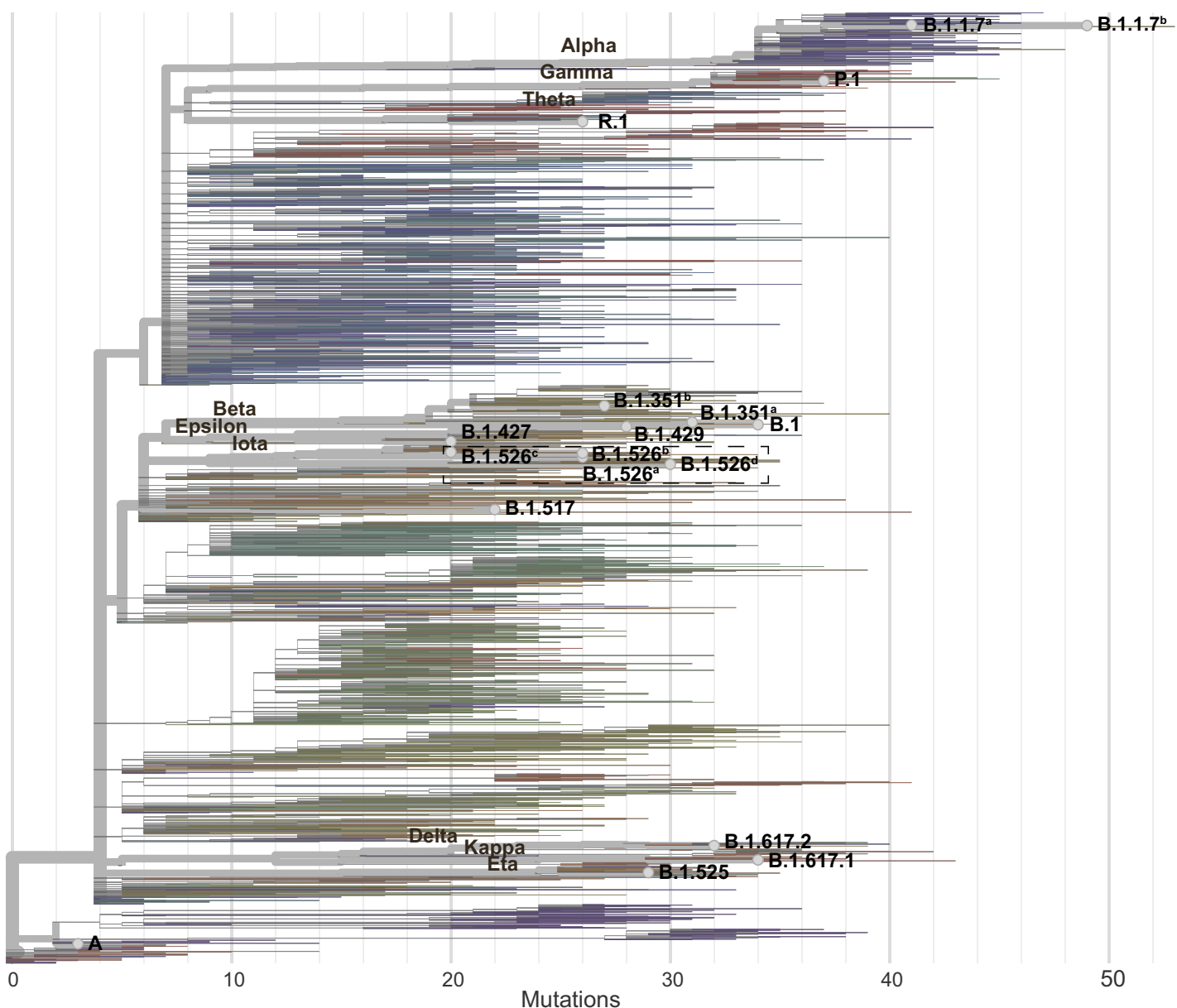

B

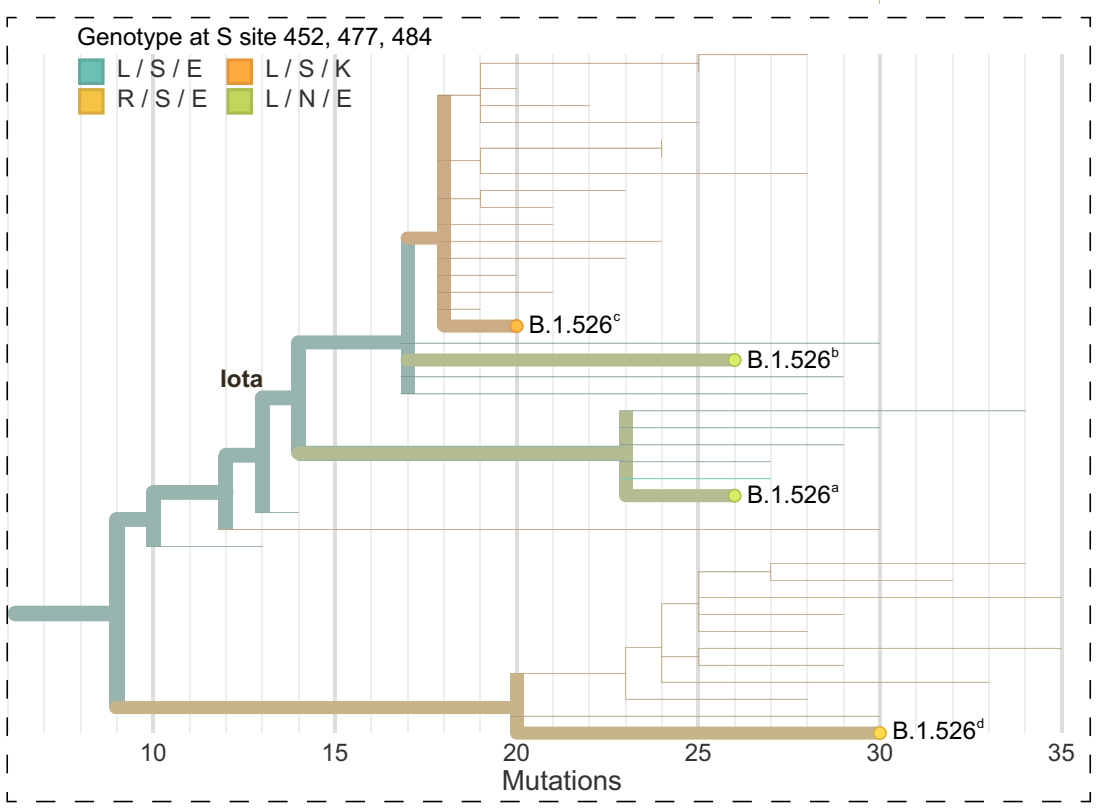

Extended Data Fig. 3 | Maximum likelihood phylogeny of SARS-CoV-2 genomes of cultured virus isolates. a, Nextclade (https://clades.nextstrain. org/) was used to generate a phylogenetic tree to show evolutionary relations between the cultured virus isolates used in this study and other publicly available SARS-CoV-2 genomes. Branches are colored by Pango Lineage, and labelled according to the WHO naming scheme. Highlighted are the cultured virus isolates used in this study. b. Enlarged section of the phylogenetic tree highlighting spike amino acid changes in the B.1.526 (iota) lineage viruses belonging to different clades. 
A)
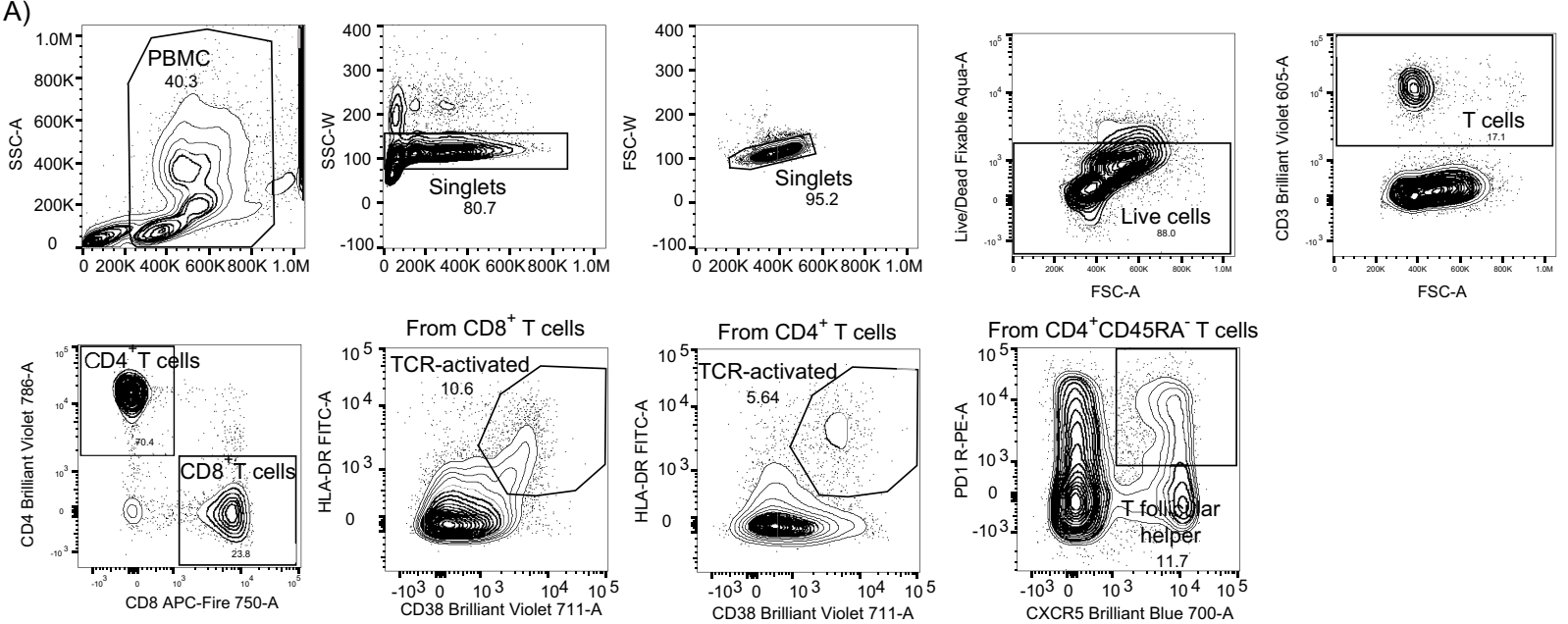

B)
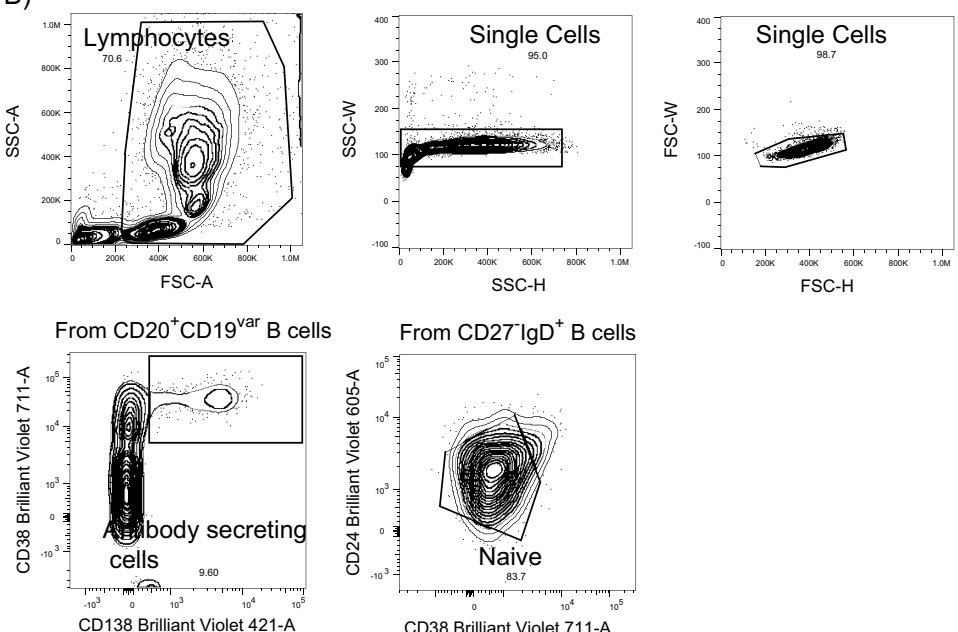

CD38 Brilliant Violet 711-A

Extended Data Fig. 4 | Gating strategies. Gating strategies are shown for the key cell populations described in Fig. 2 and Extended Data Fig. 2. a, Leukocyte gating strategy to identify lymphocytes. T cell surface staining gating strategy to identify $\mathrm{CD} 4^{+}$and $\mathrm{CD} 8^{+} \mathrm{T}$ cells, TCR-activated $\mathrm{T}$ cells and follicular $\mathrm{T}$ cells. b, B cell surface staining gating strategy to identify B cells subsets. 


\begin{tabular}{|c|c|c|c|c|c|c|c|}
\hline & & Vaccine & & Sex & & SeroStatus & \\
\hline & Age (years) & Modema & Pfizer & Female & Male & Negative & $\begin{array}{l}\text { Positive } \\
\end{array}$ \\
\hline Total Cohort \% & 45.19 & 80.56 & 19.44 & 80.56 & 19.44 & 50.0 & 50.0 \\
\hline Non- Previous exposed $\%$ & $44.28(1.833 \pm 4.242)$ & 61.11 & 38.88 & 77.7 & 83.33 & & \\
\hline Previous exposed \% & $46.11(1.833 \pm 4.242)$ & 100.0 & 0.0 & 22.22 & 16.66 & & \\
\hline
\end{tabular}

\begin{tabular}{|c|c|c|c|c|}
\hline Volunteers ID & Age & Sex & SeroStatus & Vaccine \\
\hline HCW.1.0008 & 42 & Female & Negative & Pfizer \\
\hline HCW.1.0009 & 43 & Female & Negative & Pfizer \\
\hline HCW.1.0057 & 37 & Female & Negative & Modema \\
\hline HCW.1.0074 & 49 & Female & Negative & Modema \\
\hline HOW.1.0205 & 55 & Female & Negative & Pfizer \\
\hline HCW.1.0222 & 37 & Female & Negative & Pfizer \\
\hline HCW.1.0236 & 67 & Female & Negative & Modema \\
\hline HCW.1.0288 & 42 & Female & Negative & Modema \\
\hline HOW.1.0318 & 46 & Male & Negative & Modema \\
\hline HCW.1.0422 & 29 & Female & Negative & Pfizer \\
\hline HOW.1.0447 & 44 & Female & Negative & Modema \\
\hline HCW.1.0467 & 34 & Male & Negative & Modema \\
\hline HCW.1.0578 & 56 & Male & Negative & Modema \\
\hline HCW.1.0582 & 31 & Female & Negative & Modema \\
\hline HCW.1.0596 & 61 & Female & Negative & Pfizer \\
\hline HCW.1.0607 & 39 & Female & Negative & Modema \\
\hline HCW.1.0614 & 33 & Female & Negative & Modema \\
\hline HCW.1.0636 & 52 & Male & Negative & Pfizer \\
\hline HOW.1.0023 & 59 & Female & Positive & Modema \\
\hline HCW.1.0029 & 26 & Female & Positive & Modema \\
\hline HCW.1.0090 & 56 & Female & Positive & Moderna \\
\hline HCW.1.0149 & 67 & Female & Positive & Modema \\
\hline HCW.1.0158 & 54 & Female & Positive & Modema \\
\hline HCW.1.0163 & 39 & Female & Positive & Modema \\
\hline HOW.1.0223 & 45 & Male & Positive & Modema \\
\hline HCW.1.0278 & 62 & Female & Positive & Modema \\
\hline HCW.1.0297 & 27 & Male & Positive & Modema \\
\hline HCW.1.0324 & 30 & Female & Positive & Modema \\
\hline HCW.1.0347 & 47 & Female & Positive & Modema \\
\hline HCW.1.0356 & 27 & Female & Positive & Modema \\
\hline HCW.1.0365 & 32 & Female & Positive & Modema \\
\hline HOW.1.0436 & 52 & Female & Positive & Modema \\
\hline HCW.1.0445 & 59 & Female & positive & Modema \\
\hline HCW.1.0453 & 62 & Female & Positive & Modema \\
\hline HCW.1.0605 & 58 & Female & Positive & Modema \\
\hline HCW.1.0633 & 28 & Male & Positive & Modema \\
\hline
\end{tabular}

Exact counts for each demographic category are displayed in each cell with accompanying standard deviations for each measurement. Percentages of total, where applicable, are provided in parenthesis. In cases where specific demographic information was missing, the total number of patients with complete information used for calculations is provided within the cell. 


\section{Extended Data Table 2 | Amino acid changes identified in cultured SARS-CoV-2 isolates}

\begin{tabular}{|c|c|c|c|c|c|c|c|c|c|c|c|c|c|c|c|c|c|c|}
\hline 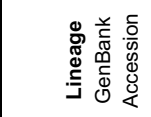 & 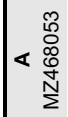 & 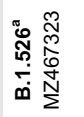 & 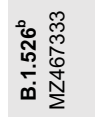 & 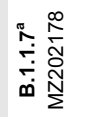 & 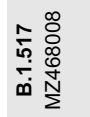 & 莡 & 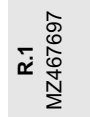 & 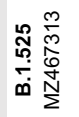 & 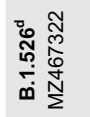 & 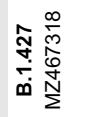 & 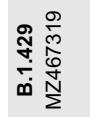 & 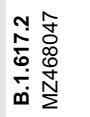 & 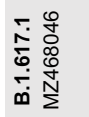 & 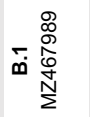 & 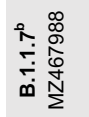 & $\bar{r} \frac{\stackrel{0}{0}}{\stackrel{N}{N}}$ & 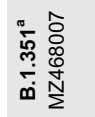 & 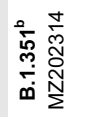 \\
\hline $\mathrm{E}$ & & & & & & & & L21F & & & & V62F & & & & & $\begin{array}{l}\text { P71L } \\
\end{array}$ & P71L \\
\hline M & & & & & & & $\begin{array}{l}\text { F28L } \\
\text { T175M }\end{array}$ & I82T & & & & 182T & $182 \mathrm{~S}$ & & & & & \\
\hline $\mathrm{N}$ & & $\begin{array}{l}\text { P13L } \\
\text { S202R } \\
\text { A414V }\end{array}$ & $\begin{array}{l}\text { P199L } \\
\text { M234I }\end{array}$ & $\begin{array}{l}\text { D3L } \\
\text { R203K } \\
\text { G204R } \\
\text { S235F }\end{array}$ & S206F & $\begin{array}{l}\text { P199L } \\
\text { M234I }\end{array}$ & $\begin{array}{l}\text { S187L } \\
\text { R203K } \\
\text { G204R } \\
\text { Q418H }\end{array}$ & $\begin{array}{l}\text { M1- } \\
\text { S2M } \\
\text { D3Y } \\
\text { A12G } \\
\text { T205I }\end{array}$ & $\begin{array}{l}\text { T205I } \\
\text { M234I }\end{array}$ & $\begin{array}{l}\text { T205I } \\
\text { T362I }\end{array}$ & $\begin{array}{l}\text { T205I } \\
\text { M234I }\end{array}$ & $\begin{array}{l}\text { D63G } \\
\text { R203M } \\
\text { D377Y }\end{array}$ & $\begin{array}{l}\text { R203M } \\
\text { D377Y }\end{array}$ & $\begin{array}{l}\text { D3Y } \\
\text { T205I }\end{array}$ & $\begin{array}{l}\text { D3L } \\
\text { R203K } \\
\text { G204R } \\
\text { S235F }\end{array}$ & $\begin{array}{l}\text { P80R } \\
\text { R203K } \\
\text { G204R }\end{array}$ & T205I & T205I \\
\hline ORF1a & & $\begin{array}{l}\text { T265I } \\
\text { T1840I } \\
\text { G1946S } \\
\text { L3201P } \\
\text { S3675- } \\
\text { G3676- } \\
\text { F3677- }\end{array}$ & $\begin{array}{l}\text { T265I } \\
\text { A1352V } \\
\text { L3201P } \\
\text { P3504S } \\
\text { S3675- } \\
\text { G3676- } \\
\text { F3677- }\end{array}$ & $\begin{array}{l}\text { T1001I } \\
\text { P1213L } \\
\text { A1708D } \\
\text { I2230T } \\
\text { M2259I } \\
\text { S3675- } \\
\text { G3676- } \\
\text { F3677- }\end{array}$ & $\begin{array}{l}\text { T265I } \\
\text { A541S } \\
\text { G989V } \\
\text { H1580Y }\end{array}$ & $\begin{array}{l}\text { T265I } \\
\text { T2977I } \\
\text { L3201P } \\
\text { S3675- } \\
\text { G3676- } \\
\text { F3677- }\end{array}$ & & $\begin{array}{l}\text { T2007I } \\
\text { L2609I } \\
\text { S3675- } \\
\text { G3676- } \\
\text { F3677- }\end{array}$ & $\begin{array}{l}\text { T265I } \\
\text { T2087I } \\
\text { K3162E } \\
\text { L3201P } \\
\text { A3209V } \\
\text { P3359S } \\
\text { S3675- } \\
\text { G3676- } \\
\text { F3677- } \\
\text { V3847I } \\
\text { L4126F }\end{array}$ & $\begin{array}{l}\text { T265I } \\
\text { S3158T }\end{array}$ & $\begin{array}{l}\text { T265I } \\
14205 \mathrm{~V}\end{array}$ & $\begin{array}{l}\text { P309L } \\
\text { A405V } \\
\text { P1640L } \\
\text { A3209V } \\
\text { V3718A }\end{array}$ & $\begin{array}{l}\text { T1567I } \\
\text { T3646A }\end{array}$ & $\begin{array}{l}\text { G150S } \\
\text { T265I } \\
\text { V649F } \\
\text { T708I } \\
\text { A1049V } \\
\text { T1854I } \\
\text { K2497N } \\
\text { R3542C } \\
\text { M4375T }\end{array}$ & $\begin{array}{l}\text { E913D } \\
\text { T1001I } \\
\text { A1306T } \\
\text { A1708D } \\
\text { P2046L } \\
\text { I2230T } \\
\text { M2259I } \\
\text { S3675- } \\
\text { G3676- } \\
\text { F3677- } \\
\text { L3736F } \\
\text { L3829F } \\
\end{array}$ & $\begin{array}{l}\text { S1188L } \\
\text { K1795Q } \\
\text { G2941S } \\
\text { S3675- } \\
\text { G3676- } \\
\text { F3677- }\end{array}$ & $\begin{array}{l}\text { T265I } \\
\text { T333M } \\
\text { Y1598C } \\
\text { K1655N } \\
\text { E1843D } \\
\text { T2174I } \\
\text { K3353R } \\
\text { S3675- } \\
\text { G3676- } \\
\text { F3677- } \\
\text { T4065I }\end{array}$ & $\begin{array}{l}\text { T265I } \\
\text { K1655N } \\
\text { K3353R } \\
\text { S3675- } \\
\text { G3676- } \\
\text { F3677- }\end{array}$ \\
\hline ORF1b & & $\begin{array}{l}\text { P314L } \\
\text { Q1011H }\end{array}$ & $\begin{array}{l}\text { P314L } \\
\text { Q1011H }\end{array}$ & $\begin{array}{l}\text { P218L } \\
\text { P314L } \\
\text { A1432V }\end{array}$ & $\begin{array}{l}\text { P314L } \\
\text { D1506N } \\
\text { P2633S }\end{array}$ & $\begin{array}{l}\text { P314L } \\
\text { Q1011H } \\
\text { R1078C }\end{array}$ & $\begin{array}{l}\text { P314L } \\
\text { G814C } \\
\text { G1362R } \\
\text { P1936H }\end{array}$ & P314F & P314L & $\begin{array}{l}\text { P314L } \\
\text { P976L } \\
\text { D1183Y }\end{array}$ & $\begin{array}{l}\text { P314L } \\
\text { D1183Y } \\
\text { G2436C }\end{array}$ & $\begin{array}{l}\text { P314L } \\
\text { G662S } \\
\text { P1000L } \\
\text { P1570L }\end{array}$ & $\begin{array}{l}\text { P314L } \\
\text { G1129C } \\
\text { A1291S } \\
\text { M1352I } \\
\text { K2310R } \\
\text { S2312A } \\
\end{array}$ & $\begin{array}{l}\text { T132I } \\
\text { P314L }\end{array}$ & $\begin{array}{l}\text { P218L } \\
\text { P314L } \\
\text { T1511I }\end{array}$ & $\begin{array}{l}\text { P314L } \\
\text { A1219S } \\
\text { E1264D }\end{array}$ & $\begin{array}{l}\text { P314L } \\
\text { T1050I } \\
\text { Y2608H }\end{array}$ & P314L \\
\hline ORF3a & & $\begin{array}{l}\mathrm{P} 42 \mathrm{~L} \\
\mathrm{Q} 57 \mathrm{H}\end{array}$ & $\begin{array}{l}\text { P42L } \\
\text { Q57H }\end{array}$ & & $\begin{array}{l}\text { Q57H } \\
\text { D210E }\end{array}$ & $\begin{array}{l}\text { P42L } \\
\text { Q57H }\end{array}$ & & S92L & $\begin{array}{l}\text { P42L } \\
\text { Q57H }\end{array}$ & $\mathrm{Q} 57 \mathrm{H}$ & $\mathrm{Q} 57 \mathrm{H}$ & S26L & $\mathrm{S} 26 \mathrm{~L}$ & $\begin{array}{l}\text { Q57H } \\
\text { P104S } \\
\text { S171L } \\
\end{array}$ & & $\begin{array}{l}\text { Q57H } \\
\text { S253P }\end{array}$ & $\begin{array}{l}\text { Q57H } \\
\text { S171L }\end{array}$ & $\begin{array}{l}\text { Q57H } \\
\text { W131L } \\
\text { S171L } \\
\end{array}$ \\
\hline ORF6 & & & & & & & & $\begin{array}{l}\text { M1- } \\
\text { F2M } \\
\end{array}$ & & & & & & & & & & \\
\hline ORF7a & & L116F & & & & & & A105S & P34S & & & $\begin{array}{l}\text { V82A } \\
\text { L116F } \\
\text { T120I } \\
\end{array}$ & $\begin{array}{l}\text { N43Y } \\
\text { V82A }\end{array}$ & & & & & V93F \\
\hline \begin{tabular}{|l|} 
ORF8 \\
\end{tabular} & L84S & T11। & T111 & $\begin{array}{l}\text { Q27* } \\
\text { R52I } \\
\text { K68* } \\
\text { Y73C } \\
\end{array}$ & E59* & T11I & & & $\begin{array}{l}\text { T11I } \\
\text { P36S } \\
\text { A51S }\end{array}$ & & V100L & $\begin{array}{l}\text { D119- } \\
\text { F120- }\end{array}$ & & E106D & $\begin{array}{l}\text { Q27 } \\
\text { R521 } \\
\text { K68* } \\
\text { Y73C } \\
\end{array}$ & E92K & I121L & R115L \\
\hline ORF9b & & P10s & T831 & & & & & $\mathrm{H} 9 \mathrm{D}$ & & & & $\mathrm{T} 60 \mathrm{~A}$ & & & & Q77E & & \\
\hline$S$ & & $\begin{array}{l}\text { L5F } \\
\text { T95I } \\
\text { D253G } \\
\text { S477N } \\
\text { D614G } \\
\text { Q957R }\end{array}$ & $\begin{array}{l}\text { L5F } \\
\text { T95I } \\
\text { D253G } \\
\text { S477N } \\
\text { D614G } \\
\text { A701V }\end{array}$ & $\begin{array}{l}\text { H69- } \\
\text { V70- } \\
\text { Y144- } \\
\text { N501Y } \\
\text { A570D } \\
\text { D614G } \\
\text { P681H } \\
\text { T716I } \\
\text { S982A } \\
\text { D1118H }\end{array}$ & $\begin{array}{l}\text { N501T } \\
\text { Q613H } \\
\text { D614G } \\
\text { G639V }\end{array}$ & $\begin{array}{l}\text { L5F } \\
\text { T95I } \\
\text { D253G } \\
\text { E484K } \\
\text { D614G } \\
\text { A701V }\end{array}$ & $\begin{array}{l}\text { W152L } \\
\text { E484K } \\
\text { K558N } \\
\text { D614G } \\
\text { G769V }\end{array}$ & $\begin{array}{l}\text { H69- } \\
\text { V70- } \\
\text { Y144- } \\
\text { Q52R } \\
\text { A67V } \\
\text { E484K } \\
\text { D614G } \\
\text { Q677H } \\
\text { F888L }\end{array}$ & $\begin{array}{l}\text { D80G } \\
\text { Y144- } \\
\text { F157S } \\
\text { L452R } \\
\text { D614G } \\
\text { T791I } \\
\text { T859N } \\
\text { D950H }\end{array}$ & $\begin{array}{l}\text { S13I } \\
\text { W152C } \\
\text { L452R } \\
\text { D614G }\end{array}$ & $\begin{array}{l}\text { S13I } \\
\text { W152C } \\
\text { L452R } \\
\text { D614G }\end{array}$ & $\begin{array}{l}\text { T19R } \\
\text { E156- } \\
\text { F157- } \\
\text { R158G } \\
\text { L452R } \\
\text { T478K } \\
\text { D614G } \\
\text { P681R } \\
\text { D950N } \\
\text { L1141W }\end{array}$ & $\begin{array}{l}\text { T95I } \\
\text { G142D } \\
\text { E154K } \\
\text { L452R } \\
\text { E484Q } \\
\text { D614G } \\
\text { P681R } \\
\text { Q1071H }\end{array}$ & $\begin{array}{l}\text { E484K } \\
\text { N501T } \\
\text { D614G }\end{array}$ & $\begin{array}{l}\text { H69- } \\
\text { V70- } \\
\text { Y144- } \\
\text { E484K } \\
\text { N501Y } \\
\text { A570D } \\
\text { D614G } \\
\text { P681H } \\
\text { T716I } \\
\text { S982A } \\
\text { D1118H }\end{array}$ & $\begin{array}{l}\text { L18F } \\
\text { T20N } \\
\text { P26S } \\
\text { D138Y } \\
\text { R190S } \\
\text { K417T } \\
\text { E484K } \\
\text { N501Y } \\
\text { D614G } \\
\text { H655Y } \\
\text { T1027I } \\
\text { V1176F }\end{array}$ & $\begin{array}{l}\text { D80A } \\
\text { D215G } \\
\text { L241- } \\
\text { L242- } \\
\text { A243- } \\
\text { K417N } \\
\text { E484K } \\
\text { N501Y } \\
\text { D614G } \\
\text { A701V }\end{array}$ & $\begin{array}{l}\text { L18F } \\
\text { D80A } \\
\text { D215G } \\
\text { L241- } \\
\text { L242- } \\
\text { A243- } \\
\text { K417N } \\
\text { E484K } \\
\text { N501Y } \\
\text { D614G } \\
\text { Q677H } \\
\text { R682W } \\
\text { A701V }\end{array}$ \\
\hline
\end{tabular}

Cultured virus isolates were resequenced and the consensus genomes were compared to the reference genome (Accession MN908947) using Nextclade (https://clades.nextstrain.org/). Letters indicate amino acids, numbers indicate amino acid positions, asterisks indicate stop codon mutations, and dashes indicate deletions. Abbreviations: $\mathrm{E}$, envelope; $\mathrm{M}$, membrane; $\mathrm{N}$, nucleocapsid; ORF, open reading frame; S, spike. 


\section{nature portfolio}

Corresponding author(s): Akiko Iwasaki

Last updated by author(s): Sep 17, 2021

\section{Reporting Summary}

Nature Portfolio wishes to improve the reproducibility of the work that we publish. This form provides structure for consistency and transparency in reporting. For further information on Nature Portfolio policies, see our Editorial Policies and the Editorial Policy Checklist.

\section{Statistics}

For all statistical analyses, confirm that the following items are present in the figure legend, table legend, main text, or Methods section.

n/a Confirmed

$\square$ \. exact sample size $(n)$ for each experimental group/condition, given as a discrete number and unit of measurement

Х $\square$ A statement on whether measurements were taken from distinct samples or whether the same sample was measured repeatedly

The statistical test(s) used AND whether they are one- or two-sided

Only common tests should be described solely by name; describe more complex techniques in the Methods section.

$\bigotimes$ A description of all covariates tested

$\square$ A description of any assumptions or corrections, such as tests of normality and adjustment for multiple comparisons

$\searrow$ A full description of the statistical parameters including central tendency (e.g. means) or other basic estimates (e.g. regression coefficient)

$\triangle$ AND variation (e.g. standard deviation) or associated estimates of uncertainty (e.g. confidence intervals)

$\varnothing$ For null hypothesis testing, the test statistic (e.g. $F, t, r$ ) with confidence intervals, effect sizes, degrees of freedom and $P$ value noted

Give $P$ values as exact values whenever suitable.

Х $\square$ For Bayesian analysis, information on the choice of priors and Markov chain Monte Carlo settings

$\square$ For hierarchical and complex designs, identification of the appropriate level for tests and full reporting of outcomes

$\bigotimes \square$ Estimates of effect sizes (e.g. Cohen's $d$, Pearson's $r$ ), indicating how they were calculated

Our web collection on statistics for biologists contains articles on many of the points above.

\section{Software and code}

Policy information about availability of computer code

Data collection EPIC EHR software (2020 May released) (retrospective EMR review and clinical data aggregation) and REDCap 9.3.6 (clinical data aggregation).

Data analysis Jmp Pro 15.0.0 (SAS Institute) (graphs/statistics), GraphPad Prism 8.4.3(graphs/statistics), FlowJo software version 10.6 software (Tree Star), R 3.4.3 or 4.0.1 (graphs/statistics), iVar version 1.3.1 (data analysis), and Nextclade v.1.5.0 (data analysis).

For manuscripts utilizing custom algorithms or software that are central to the research but not yet described in published literature, software must be made available to editors and reviewers. We strongly encourage code deposition in a community repository (e.g. GitHub). See the Nature Portfolio guidelines for submitting code \& software for further information.

\section{Data}

\section{Policy information about availability of data}

All manuscripts must include a data availability statement. This statement should provide the following information, where applicable:

- Accession codes, unique identifiers, or web links for publicly available datasets

- A description of any restrictions on data availability

- For clinical datasets or third party data, please ensure that the statement adheres to our policy

The data generated during the current study is available: raw data is available at the source data files; genomes of SARS-CoV-2 isolates are uploaded to GenBank and accession numbers are provided in the supplement and the aligned consensus genomes are available on GitHub (https://github.com/grubaughlab/ paper_2021_Nab-variants). 


\section{Field-specific reporting}

Please select the one below that is the best fit for your research. If you are not sure, read the appropriate sections before making your selection. $\bigotimes$ Life sciences $\quad \square$ Behavioural \& social sciences $\quad \square$ Ecological, evolutionary \& environmental sciences

For a reference copy of the document with all sections, see nature.com/documents/nr-reporting-summary-flat.pdf

\section{Life sciences study design}

All studies must disclose on these points even when the disclosure is negative.

Sample size

No statistical methods were used to calculate the sample size. Sample size was determined based on the number of adults health care workers ( $\geq 18$ years old) from the Yale-New Haven Hospital (YNHH) that received the mRNA vaccine (Moderna or Pfizer) between November 2020 and January 2021, and were recruited and consented with the current study. This study enrolled 40 volunteers under IRB and HIC approved protocol \#2000028599. The sample size number was sufficient once it kept errors at an acceptably low levels. Informed consent was obtained by trained staff and sample collection commenced immediately upon study enrollment. HCWs were followed serially postvaccination. Clinical specimens were collected were collected at baseline (previous to vaccination), 7- and 28- post first vaccination dose, and 7-, 28- and 70-days post second vaccination dose. Sixteen SARS-CoV-2 isolates belonging to 12 lineages, were selected from the Yale SARSCoV-2 Genomic Surveillance Initiative's weekly surveillance program in Connecticut, US. The sixteen isolates represent variants of concern/ interest, lineages with mutations of concern/interest, and controls that were identified within the surveillance program.

Data exclusions One participant that received an adenovirus- based vaccine was excluded from this study. For the current study we had only included participants that received mRNA vaccines.

Replication Neutralization assays were done in duplicate with 6 fold dilution for each sample. ELISAs were done in duplicate with 4 fold dilutions for each samples. Replications were successful. The flow cytometry findings were not replicated due to samples availability limitations, however longitudinal analyses were performed from human individuals.

Randomization Vaccinated donors were stratified in two major groups, previously infected with SARS-CoV2 (recovered) on uninfected (naive), confirmed by RT-qPCR and serology.

Blinding The clinical data were collected using EPIC EHR May 2020 and REDCap 9.3.6 software. Blood acquisition was performed and recorded by a separate team. Vaccinated HCW's clinical information and time points of collection information was not available until after processing and analyzing raw data by flow cytometry and ELISA. ELISA, neutralizations, and flow cytometry analyses were blinded.

\section{Reporting for specific materials, systems and methods}

We require information from authors about some types of materials, experimental systems and methods used in many studies. Here, indicate whether each material, system or method listed is relevant to your study. If you are not sure if a list item applies to your research, read the appropriate section before selecting a response.

Materials \& experimental systems

\begin{tabular}{l|l}
\hline$n / a$ & Involved in the study \\
\hline & $\bigotimes$ Antibodies \\
$\square$ & $\square$ Eukaryotic cell lines \\
$\square$ Palaeontology and archaeology \\
$\square$ \\
$\square$ Animals and other organisms \\
$\square$ \\
$\square$ Clinical data
\end{tabular}

\begin{tabular}{l|l}
\multicolumn{2}{l}{ Methods } \\
\hline n/a & Involved in the study \\
$\square$ & $\square$ ChIP-seq \\
$\square$ & $\bigotimes$ Flow cytometry \\
$\square$ & $\square$ MRI-based neuroimaging
\end{tabular}

\section{Antibodies}

Antibodies used

All antibodies used in this study are against human proteins. BB515 anti-hHLA-DR (G46-6) (1:400) (BD Biosciences \# 564516), BV605 anti-hCD3 (UCHT1) (1:300) (BioLegend \#300460), BV785 anti-hCD19 (SJ25C1) (1:300) (BD Biosciences \# 363028), BV785 anti-hCD4 (SK3) (1:200) (BioLegend \# 344642), APCFire750 or BV711 anti-hCD8 (SK1) (1:200) (BioLegend \# 344746), AlexaFluor 700 antihCD45RA (HI100) (1:200) (BD Biosciences \# 560673), PE anti-hPD1 (EH12.2H7) (1:200) (BioLegend \# 621608), APC or PE-CF594 antihTIM3 (F38-2E2) (1:50) (BioLegend \# 345012), BV711 anti-hCD38 (HIT2) (1:200) (BioLegend \# 303528), BB700 anti-hCXCR5 (RF8B2) (1:50) (BD Biosciences \# 566470), PE-CF594 anti-hCD25 (BC96) (1:200) (BD Biosciences \#562403), AlexaFluor 700 anti-hTNFa (MAb11) (1:100) (BioLegend \# 506338), PE or APC/Fire750 anti-hIFNy (4S.B3) (1:60) (BioLegend \# 343536), FITC anti-hGranzymeB (GB11) (1:200) (BioLegend \# 515403), BV785 anti-hCD19 (SJ25C1) (1:300) (BioLegend \# 302240), BV421 anti-hCD138 (MI15) (1:300) (BioLegend \# 356516), AlexaFluor700 anti-hCD20 (2H7) (1:200) (BioLegend \# 302310), AlexaFluor 647 anti-hCD27 (M-T271) (1:350) (BioLegend \# 356434), PE/Dazzle594 anti-hlgD (IA6-2) (1:400) (BioLegend \# 348240), Percp/Cy5.5 anti-hCD137 (4B4-1) (1:150) (BioLegend \#309814) and PE anti-CD69 (FN-50) (1:200) (BioLegend \# 310906), APC anti-hCD4OL (24-31) (1:100) (BioLegend \# 
313008), HRP anti-Human IgG Antibody (\#A00166) (GenScript) (1:5,000), SARS-CoV-2 Human Anti-Spike (AM006415) (1:500) (Active Motif \#91351), SARS-CoV-2 Human anti-Nucleocapsid (1A6) (1:500) (Active Motif \# MA5-35941).

Validation

All antibodies used in this study are commercially available, and all have been validated by the manufacturers and used by other publications. All antibodies listed below used for flow cytometry were quality control tested by immunofluorescent staining with flow cytometric analysis. HRP anti-Human IgG Antibody (\#A00166) (GenScript), SARS-CoV-2 Human Anti-Spike (AM006415) (Active Motif \#91351), SARS-CoV-2 Human anti-Nucleocapsid (AM006415) (Active Motif \#91351) were quality control tested by ELISA. Likewise, we titrated these antibodies according to our own our staining conditions. The following were validated in the following species: BB515 anti-hHLA-DR (G46-6) (BD Biosciences) (Human, Rhesus, Cynomolgus, Baboon), BV605 anti-hCD3 (UCHT1) (BioLegend) (Human, Chimpanzee), BV785 anti-hCD19 (SJ25C1) (BD Biosciences) (Human), BV785 anti-hCD4 (SK3) (BioLegend) (Human), APCFire750 or PECy7 or BV711 anti-hCD8 (SK1) (BioLegend) (Human, Cross-Reactivity: African Green, Chimpanzee, Cynomolgus, Pigtailed Macaque, Rhesus, Sooty Mangabey), AlexaFluor 700 anti-hCD45RA (HI100) (BD Biosciences) (Human), PE anti-hPD1 (EH12.2H7) (BioLegend) (Human, African Green, Baboon, Chimpanzee, Common Marmoset, Cynomolgus, Rhesus, Squirrel Monkey), APC anti-hTIM3 (F38-2E2) (BioLegend) (Human), BV711 anti-hCD38 (HIT2) (BioLegend) (Human, Chimpanzee, Horse), BB700 anti-hCXCR5 (RF8B2) (BD Biosciences) (Human), PE-CF594 anti-hCD25 (BC96) (BD Biosciences) (Human, Rhesus, Cynomolgus, Baboon), AlexaFluor 700 antihTNFa (MAb11) (BioLegend) (Human, Cat, Cross-Reactivity: Chimpanzee, Baboon, Cynomolgus, Rhesus, Pigtailed Macaque, Sooty Mangabey, Swine), PE or APC/Fire750 anti-hIFNy (4S.B3) (BioLegend) (Human, Cross-Reactivity: Chimpanzee, Baboon, Cynomolgus, Rhesus), FITC anti-hGranzymeB (GB11) (BioLegend) (Human, Mouse, Cross-Reactivity: Rat), BV785 anti-hCD19 (SJ25C1) (BioLegend) (Human), BV421 anti-hCD138 (MI15) (BioLegend) (Human), AlexaFluor700 anti-hCD20 (2H7) (BioLegend) (Human, Baboon, Capuchin Monkey, Chimpanzee, Cynomolgus, Pigtailed Macaque, Rhesus, Squirrel Monkey), AlexaFluor 647 anti-hCD27 (M-T271) (BioLegend) (Human, Cross-Reacitivity: Baboon, Cynomolgus, Rhesus), PE/Dazzle594 anti-hlgD (IA6-2) (BioLegend) (Human), Percp/Cy5.5 antihCD137 (4B4-1) (BioLegend), Human, Cross-Reactivity: Chimpanzee, Baboon, Cynomolgus, Rhesus), PE anti-CD69 (FN-50) (BioLegend) (Human, African Green, Baboon, Chimpanzee, Cynomolgus, Pigtailed Macaque, Rhesus), APC anti-hCD40L (24-31) (BioLegend) (Human, African Green, Baboon, Cynomolgus, Rhesus), HRP anti-Human IgG Antibody (\#A00166) (GenScript) (Human), SARS-CoV-2 Human Anti-Spike (AM006415) (Active Motif) (Human), SARS-CoV-2 Human anti-Nucleocapsid (1A6) (Active Motif) (Human).

\section{Eukaryotic cell lines}

Policy information about cell lines

Cell line source(s)

TMPRSS2-VeroE6 kidney epithelial cell line was obtained from the ATCC

Authentication

TMPRSS2-VeroE6 was obtained from ATCC, tested and authenticated by morphology, karyotyping, and PCR based approaches.

Mycoplasma contamination

The cell line tested negative for contamination with mycoplasma.

Commonly misidentified lines

(See ICLAC register)

No commonly misidentified cell lines were used in the study.

\section{Human research participants}

Policy information about studies involving human research participants

Population characteristics

Cohort characteristics: age (average, 46.19), sex (Male 19.44\% / Females 80.56.\%). Full demographic data is included in Extended data table 1.

Recruitment

HCW volunteers from the Yale New Haven Hospital (YNHH) were recruited between November 2020 and January 2021 during SARS-CoV-2 vaccination program. Participants were enrolled during the vaccination program with no self selection. The study goal (charactherization of the immune response post vaccination) was explained to the participants. Participants interested in the study, consented with the current study and were recruited. Informed consent was obtained by trained staff and sample collection commenced immediately upon study enrollment. Plasma and PBMCs samples were collected at baseline (previous to vaccination), 7- and 28- post first vaccination dose, and 7-, 28- and 70-days post second vaccination dose.

Ethics oversight

Yale Human Research Protection Program Institutional Review Boards. Informed consents were obtained from all enrolled healthcare workers. - Our research protocol was reviewed and approved by the Yale School of Medicine IRB and HIC (\#2000028599). Informed consent was obtained by trained staff and records maintained in our research database for the duration of our study. There were no minors included on this study. 


\section{Plots}

Confirm that:

邓 The axis labels state the marker and fluorochrome used (e.g. CD4-FITC).

Х The axis scales are clearly visible. Include numbers along axes only for bottom left plot of group (a 'group' is an analysis of identical markers).

$\bigotimes$ All plots are contour plots with outliers or pseudocolor plots.

$\bigotimes$ A numerical value for number of cells or percentage (with statistics) is provided.

\section{Methodology}

Sample preparation

Frozen isolated PBMCs were stained for live and dead markers, blocked with Human TruStan FcX, stained for surface markers and then fixed with PFA 4\%. For intracellular cytokine staining following stimulation, cells were surface stained, washed and fixed in 4\% PFA. After permeabilization with $1 \mathrm{X}$ Permeabilization Buffer cells were stained for intracellular cytokines analysis.

Instrument

Cells were acquired on an Attune NXT (ThermoFisher).

Software

Data were analysed using FlowJo software version 10.6 software (Tree Star).

Cell population abundance

Cell population abundance: Cells populations were reported in various formats including proportion of live, single PBMC (\% of Live), or as a proportion of a parent gate (\% of CD4 T cells, \% of Monocytes, etc.). The full gating path for clarification is included in the extended figure 4.

Gating strategy

SSC-A and FSC-A parameters were used to select leukocytes from isolated PBMCs. Live and dead cells were defined based on aqua staining. Singlets were separated based on SSC/ FSC parameters. Leukocytes were gated based on to identify lymphocytes (CD3/CD4/CD8/CD19 markers). Activated T cells were defined using HLA-DR, CD38, CCR7,CD127, PD1, TIM-3, CXCR5, CD45RA, CD25.

\Tick this box to confirm that a figure exemplifying the gating strategy is provided in the Supplementary Information. 